\title{
Note on dimension theory for metric spaces *
}

\author{
by \\ J. Nagata (Osaka, Japan)
}

Recentiy, a dimension theory for general metric spaces has been ostablished by M. Katětov and by K. Morita (see [4] and [5]) independently. They have extended the stum, decomposition and product theorems to non-separable metric spaces and have shown the eqnivalence of the Lebesgue dimension and the inductive dimension $\left({ }^{\mathbf{1}}\right)$. On the other hand, the following theorem of $P$. Alexandroff and $P$. Urysohn is well known:

In order that a $T_{1}$-topological space $R$ be metrizable it is necessary and sufficient that there exists a sequence $\mathfrak{B}_{1}>\mathfrak{V}_{2}^{*}>\mathfrak{B}_{2}>\mathfrak{B}_{3}^{*}>\ldots$ of open coverings such that $\left.\left\{S\left(p, \mathfrak{D}_{m}\right) \mid m=1,2, \ldots\right\}{ }^{2}\right)$ is a nbd (neighbourhood) basis for each point $p$ of $R$.

The purpose of the present note is to refine this theorem to a theorem concerning $n$-dimensionality of metric spaces and to develop the dimension theory for general metric spaces. In $\$ 1$ we shail prove that Alexandroff-Urysohn's theorem turns into a theorem asserting a necessary and sufficient condition for $n$-dimensionality if we add the condition order $\mathfrak{V}_{m} \leqslant n+1(n=1,2, \ldots)$ to the original condition. Furthermore, concerning that theorem it will be shown that we may replace order $\mathfrak{W}_{m} \leqslant n+1$ by Alexandroff-Kolmogoroff's length of $\mathfrak{W}_{m} \leqslant n+1$ (see [1]). In $\S 2$ we shall apply the result of $\S 1$ to the study of the connections between dimension and metric function. $\$ 3$ contains applications of the result of $\$ 1$ to the embedding of $n$-dimensional metric spaces into products of 1 -dimensional spaces. The final section is deroted to

* Tho content of this paper is a development in detail of our brief notes pub. lished in l'roc. of Trpan Acad. 32 (1956).

(1) ind $\operatorname{dim} \varnothing=-1$ for a vacuous set $\emptyset$, and ind $\operatorname{dim} R \leqslant n$ if and only if for any pair of a closed set $F$ and an open set $G$ with $F \subseteq G$ there exists an open set $U$ such that $F \subseteq U \subseteq G, \operatorname{dim} B(U) \leqslant n-1$, where we denote by $B(U)$ the boundary of $D$.

(8) $S(p, D)=\bigcup\{V \mid p \in V \in \mathscr{V}\}$ for a covering $\mathfrak{V}$ of $R, S(A, \mathscr{D})=\cup\{V \mid \nabla \cap A \neq \emptyset$, $V \in 2$ J for a subset $A$ of $R, V^{*}=\{S(V, \mathscr{V}) \mid P \in \mathscr{V}\}$. $\mathscr{V}$ is oalled a star-refinement of $\mathcal{U}$ if $2^{*}<$ ג. The notation of this paper is chiefly due to [8]. See also [2] with respect to the notions. 
the embedding of $n$-dimensional metric spaces into a product of Euclidean $(2 n+1)$-space with a zero-dimensional space and to its modifications.

Throughout this paper all spaces are metric or metrizable, and all coverings are open, unless the contrary is explicitily statiod.

\section{\$1. The main theorem.}

Definition. For two collections $\mathfrak{X}, \mathfrak{U}^{\prime}$ of open sets we donote by $\mathfrak{U}<\mathfrak{U}^{\prime}$ the fact that $U \subseteq U^{\prime}$ for every $U \in \mathfrak{U}$ and for some $U^{\prime} \in \mathfrak{U}^{\prime}$,

Derintrios. We mean by a disjointed collection a collection $\mathfrak{U}$ of open sets such that $U, U^{\prime} \in \mathfrak{U}$ and $U \neq U^{\prime}$ imply $U \cdots, U^{\prime}-\varnothing$.

Theorsa 1. In order that $\operatorname{dim} R \leqslant n$ for a metric space $R$ it is necessary and sufficient that there exist $n+1$ sequences $\mathfrak{u}_{1}^{i}, \mathfrak{x}_{2}^{i}>\ldots$ $(i=1,2, \ldots, n+1)$ of disjointed collections suoh that $\left\{\mathfrak{r}_{m}^{t} \mid i \ldots, 1, \ldots, n+\ldots\right.$ $m=1,2, \ldots\}$ is an open basis of $R$.

Proof. If $\operatorname{dim} R=0\left({ }^{3}\right)$, then by [5] there exists a seaquence $\mathfrak{B}_{m}$ $(m=1,2, \ldots)$ of locally finite coverings ( $\left.{ }^{4}\right)$ consisting of open and closed sets such that $S\left(p, \mathfrak{B}_{m}^{\Gamma}\right)(m=1,2, \ldots)$ is a ubd basis of each point $p$ of $R$. Let $\mathfrak{B}_{m}=\left\{\mathbb{V}_{a} \mid a<\tau\right\}$, then we define a sequence of coverings by

and

$$
\mathfrak{P}_{m}^{\prime}=\left\{V_{\mathrm{a}}-\bigcup_{\beta} \cup V_{\beta} \mid \alpha<\tau\right\}
$$

$$
\mathfrak{U}_{1}=\mathfrak{B}_{1}^{\prime}, \quad \mathfrak{U}_{2}=\mathfrak{U}_{1} \wedge \mathfrak{B}_{2}^{\prime}, \quad \mathfrak{U}_{3}:-\mathfrak{U}_{2} \wedge \mathfrak{B}_{3}^{\prime}, \ldots
$$

It is clear that $\mathfrak{U}_{1}>\mathfrak{U}_{2}>\ldots$ is a sequence of disjointed collections, and $\left\{\mathfrak{U}_{m} \mid m=1,2, \ldots\right\}$ is an open basis of $R$.

Conversely, if there exists a sequence $\mathfrak{H}_{1}>\mathfrak{H}_{2}>\ldots$ of disjointed collections such that $\left\{\mathfrak{u}_{m} \mid m=1,2, \ldots\right\}$ is an open basis of $R$, then for an arbitrary point $p$ of $R, p \in U \in \mathfrak{U}_{m}$ implies

$$
U \cap U^{\prime}=\emptyset \text { for } U^{\prime} \text { with } U: \neq U^{\prime} \in \mathfrak{H}_{m},
$$

and $p \notin U$ for every $U \in \mathfrak{U}_{m}$ implies

$$
\cap\left\{S\left(p, \mathfrak{H}_{j}\right) \mid j=1, \ldots, m \cdots 1 ; S\left(p, \mathfrak{H}_{j}\right) \neq\left(\bigotimes_{\}}=: p\right.\right.
$$

by the fact that $\left\{\mathfrak{U}_{m} ! m=1,2, \ldots\right\}$ is an open basis of $R$, and $\mathfrak{L l}_{m \rightarrow}>\mathfrak{U}_{m+1}>\ldots$ Hence each $\mathfrak{H}_{m}$ is locally finite and consists of opon and closed sotis, and hence $\operatorname{dim} R=0$ follows from $[;, 5]$.

Now we proceed to $n$-dimensional cases. Let $\operatorname{dim} l 2 \leqslant n$; then we can decompose $R$ into $n+1 \quad 0$-dimonsional spaces $R_{i}(i=1, \ldots, n+1)$

(9) From now on wo grsume $R \neq \varnothing$.

(4) We call 2 a locally finite covering if every point of $k$ hus somo nbd interweoting only finitely many eloments of $v$. by the genemel decomposition theorem due to Katetor and to Moritia, i.e.,

$$
R=\bigcup_{i=1}^{i n+1} R_{i}, \quad \operatorname{dim} R_{i}=0 .
$$

Then there exists a sequence $\mathfrak{B}_{1}^{i}>3_{2}^{i}>\ldots$ of disjointed collections of $R_{i}$ such that $\left\{\mathfrak{i}_{m}^{i} \mid m=1,2, \ldots\right\}$ is an open basis of $R_{i}$. As is obvious from the above discussion for 0 -dimensional cases, we may assume that every $\mathfrak{O}_{i}^{m}$ covers $X_{i}$. Wo put $\mathfrak{B}_{m}^{i}=\left\{V_{a m} \mid \alpha \in A\right\}$ and take the maximal positive number $\varepsilon$ for each $x \in V_{\text {utm }}$ such that $S_{e}(x) \cap R_{i} \subset V_{a m}{ }^{(5)}$. Furthermore we defino

$$
\begin{gathered}
\varepsilon(m, x) \cdots \min (1 / m, \varepsilon / 2), \quad U_{u m}=\bigcup\left\{\left\{S_{r(m, x)}(x) \mid x \in V_{u m}\right\},\right. \\
\left\langle\mathfrak{r}_{m}^{i} \cdots\left\{U_{u m} \mid \alpha \in A\right\} .\right.
\end{gathered}
$$

Then it aasily follows from $\mathfrak{Q}_{1}^{t}>\mathfrak{Y}_{2}^{i} \cdots \ldots$ and from the disjointedness of $\mathfrak{Y}_{m}^{i}$ that $\mathfrak{U}_{1}^{i}>\mathfrak{H}_{2}^{i}>\ldots$ and oach $\mathfrak{U}_{m}^{i}$ is a disjointed collection. Next we take an arbitrary point $x$ of $R$ and a positive number $\delta$. We can select positive integers $m, l$ such that

$$
2 / m<\delta, \quad l>m, \quad x \in V_{a l} \subset S_{1 / m}(x) \text { for some } V_{a l} \in \mathfrak{B}_{l}^{i} .
$$

since for these integors

$$
x \in U_{a l} \subset s_{2 / m}(x) \subset s_{s}(x)
$$

is obvious, it follows that $\left\{\mathfrak{r}_{m}^{t} \mid i=1, \ldots, n+1 ; m=1,2, \ldots\right\}$ is an open basis of $R$.

Conversoly, if $R$ admits $n+1$ sequences $\mathfrak{H}_{1}^{i}>\mathfrak{H}_{2}^{i}>\ldots(i=1, \ldots, n+1)$ such that $\left\{\mathfrak{I}_{m}^{i} \mid i=1, \ldots, n+1 ; m=1,2, \ldots\right\}$ is an open basis of $R$, then we define $n+1$ subspaces $R_{i}$ of $R$ by

$$
R_{i}=\left\{x \mid S\left(x, \mathfrak{X}_{m}^{i}\right)(m=1,2, \ldots) \text { is a nbd basis of } x\right\} \text {. }
$$

Considering $\mathfrak{H}_{m}^{i}$ a disjointed collection of $R_{t},\left\{\mathfrak{l}_{m_{t}}^{i} m=1,2, \ldots\right\}$ is an open busis of $R_{l}$; henes dim $R_{i}=0$ follows from the 0 -dimensional ease.

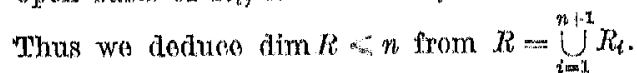

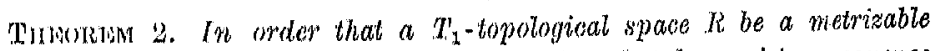
space with $\operatorname{dim} R$. n it is nocessary and sufficient that there exists a sequenoe

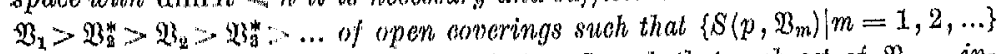
is a nbd basis for wash point $p$ of $R$ and such that each set of $\mathfrak{B}_{m+1}$ intersects at most $n+1$ sets of $\mathfrak{B}_{m}$.

(8) $x_{B}(x) \cdots\{y \mid e(x, y) \ldots$ the distunce betwoend $x$ and $y<\varepsilon\}$ 
Proof. Necessity. If $R$ is a motric spaco with $\operatorname{dim} R \therefore n$, then by the general decomposition theorem $R=: \int_{i=1}^{n+1} R_{i}$ for some 0 -dimensional spaces $R_{i}(i=1, \ldots, n+1)$.

(A) Let $\mathfrak{H}=\left\{U_{n} \mid \alpha \in A\right\}$ be an arbitrary locally finito sovering of $R$; then there exists a disjointed covering $\mathfrak{P}_{i}=\left\{W_{a} \mid \alpha \in A\right\}$ of $R_{i}$ such that $\nabla_{u} \subset U_{u}$. By putting

such that

$$
\begin{aligned}
& V_{a}^{\prime}=\bigcup\left\{S_{e(x) / 2(x)}(x) \mid x \in V_{a}^{\prime}\right\} \quad \text { for } \quad e(x)>0 \\
& R_{i} \cap S_{e(x)}(x) \subset V_{u}, \quad S_{r(x)}(m) \subset U_{u},
\end{aligned}
$$

we get a disjointed collection $\mathfrak{W}_{i}=\left\{V_{a}^{\prime} \mid \alpha \in \mathcal{A}\right\}$ of $R$ sucth thut $\mathfrak{Y}_{i}<\mathfrak{L}$. Hence $\mathfrak{B}^{\prime}=\bigcup_{i=1}^{n+1} \mathfrak{B}_{i}^{\prime}$ is a locally finite covoring of $k$ of order $x^{\prime} \mu_{1}+1$ and is a refinement of $\mathfrak{X}\left({ }^{\circ}\right)$. Hence there exists an open covering $\mathfrak{V}^{\prime \prime}: \ldots\left\{V_{\beta}^{\prime \prime} \mid \beta \in B\right\}$ of $R$ such that $\bar{V}_{\beta}^{\prime \prime} \subseteq V_{A}^{\prime}$ for $\mathfrak{B}^{\prime}=\left\{V_{\beta}^{\prime} \mid \beta \in \mathcal{B}\right\}$. It is owsily suen from order $\mathfrak{B}^{\prime} \leqslant n+1$ and from the property of $\mathfrak{B}^{\prime \prime}$ that every point $p$ of $R$ has some nbd intersecting at most $n+1$ of the sets bolonging to $\$ Z^{\prime \prime}$; we call such a covering to be of local order $\leqslant n+1$.

Now let $\mathfrak{U}_{1}>\mathfrak{U}_{2}>\ldots$ be a sequence of coverings of $R$ streh that; $\left\{S\left(p, \mathfrak{H}_{m}\right) \mid m=1,2, \ldots\right\}$ is a nbd basis for each point $p$, then from the paracompactness (7) of $R$ we may assume that all $\mathfrak{H}_{m}$ are locally finitio. Hence from (A) we get a refinement $\mathfrak{B}_{1}$ of $\mathfrak{H}_{1}$ such that the local order of $\mathfrak{H}_{1} \leqslant n+1$. Furthermore, we ean select locally finite coverings $\mathfrak{B}, \mathfrak{Q}$ such that $\mathfrak{P}^{*}<\mathfrak{B}_{1}$ and such that every set of $\mathfrak{Q}$ intersects at most $n+1$ sets of $\mathfrak{V}_{1}$. Since $\mathfrak{U}_{2} \wedge \Re \wedge \mathfrak{Q}$ is locally finite, from (A) wo obtain a refinement $\mathfrak{B}_{2}$ of $\mathfrak{U}_{2} \wedge \mathfrak{P} \wedge \mathfrak{Q}$ with the local order of $\mathfrak{B}_{2} \leqslant n+1$. Then it follows clearly that $\mathfrak{B}_{2}<\mathfrak{H}_{2}, \mathfrak{B}_{2}^{*}<\mathfrak{B}_{1}$ and each set of " $\mathfrak{B}_{2}$ intersects at most $n+1$ sets of $\mathfrak{B}_{1}$. By repeating such processes we obtain a sequence $\mathfrak{B}_{1}>\mathfrak{B}_{2}^{*}>\mathfrak{B}_{2}>\mathfrak{B}_{3}^{*}>\ldots$ of open coverings such that $\mathfrak{B}_{m}<\mathfrak{H}_{m}$ and such that each set of $\mathfrak{B}_{m+1}$ intersects at most $n+1$ of the sets belonging to $\mathfrak{B}_{m}$. Since $\left\{S\left(p, \mathfrak{U}_{m}\right) \mid m=1,2, \ldots\right\}$ is a nbd basis of $p,\left\{S\left(p, \mathfrak{B}_{m}\right) \mid m=1,2, \ldots\right\}$ is also a nbd basis of $p$, and hence the necessity is proved.

Sufficiency. The metrizability of such a space is obvious from Urysohn-Alexandroff's theorem. We divide the proot' of $n$-dimensionality into three parts.

(") We call $\mathfrak{V}^{\prime}$ a refinement of $\mathfrak{U}$ if $\mathfrak{V}^{\prime}<\mathfrak{U}$, $i$. e., for overy $Y$ " $\mathfrak{V}^{\prime}$ there existe $U \in \mathfrak{U}$ with $U \supset \nabla$.

$\left({ }^{7}\right)$ Every fully normal space is paracompact by [6]. $R$ is called paracornptuct il: every oovering of $R$ has a locally finite refinement, and it is called fally normal if every covering has a star-refinement. It is well known that every metric space is
1. If $\mathfrak{B}_{1}>\mathfrak{B}_{2}^{*}>\ldots$ is a sequence satisfying the condition of this proposition, then it is easily seen that for each point $p$ of $R$ $S^{n+2}\left(p, \mathfrak{B}_{m++1+n+2}\right)\left(^{8}\right)$ is contained in some set of $\mathfrak{B}_{m+1}$. Therefore each $S^{n+2}\left(p, \mathfrak{S}_{m+1+n+2}\right)$ intersects at most $n+1$ sets of $\mathfrak{B}_{m}$. Putting

$$
\mathfrak{U}_{n}=\mathfrak{V}_{1+(m-1)(n+3)} \quad(m=1,2, \ldots),
$$

we get a sequence $\mathfrak{U}_{1}>\mathfrak{I}_{2}^{*}>\mathfrak{I}_{2}>\mathfrak{U}_{3}^{*}>\ldots$ of open coverings such that $\left\{S\left(p, \mathfrak{H F}_{m}\right) \mid m=1,2, \ldots\right\}$ is a nbd basis of $p \in R$ and such that each $S^{n+2}\left(p, \mathfrak{K}_{m \cdots+1}\right)$ intersects at most $n+1$ sets of $\mathfrak{U}_{m}$.

Let $\mathfrak{U}_{m}=\left\{U_{a} \mid a<\tau\right\}$; then we (an prove first that there exist open sets $V_{u}^{i}$ stuch that,

and such that

$$
\sum_{i=1}^{n+1} U_{a}^{i} \subset U_{a}, \quad U_{a}^{i}, \quad U_{\beta}^{i}=0 \text { for } \quad a \neq \beta
$$

$$
U_{u} \supseteq M \in \mathfrak{U}_{m+1} \text { implies } \quad H \subset U_{a}^{i} \text { for some } U_{a}^{i} .
$$

To prove this we define $U_{u 2}^{i}(a<\tau)$ by induction such that

$$
\begin{aligned}
& \text { 1) } \bigcup_{i=1}^{n+1} U_{\alpha} \subseteq U_{u} \\
& \text { 2) } U_{a}^{i} \cap U_{\beta}^{i}=\varnothing \text { for } \beta<\alpha \text {, } \\
& \text { 3) } U_{a} \supseteq M I \in \mathfrak{U}_{m+1} \text { implies } M \subset U_{a}^{i} \text { for some } U_{a}^{i} \text {, } \\
& \text { 4) } U_{a}^{i} \cap W_{u}^{n-i+2}=\varnothing \quad(i=1, \ldots, n+1),
\end{aligned}
$$

where we put $s_{a}^{k}=\left\{p \mid S^{k}\left(p, \mathfrak{L}_{n+1}\right)\right.$ intersects some $k$ sets of $\left.U_{\gamma}(\gamma>\alpha)\right\}$ $(k=1, \ldots, n+1)$ and $W_{u}^{k=}=S_{a}^{k} \cup S_{u}^{k+1} \cup \ldots \cup S_{a}^{n+1}$.

For $x=0$ we define

$$
U_{0}^{1}=U_{0}, \quad U_{0}^{i}=\emptyset \quad(i=2, \ldots, n+1) .
$$

Since $S\left(p, \mathfrak{H}_{m+1}\right)$ intersects at most $n+1$ of $U_{u}(\alpha<\tau), U_{0}^{1} \cap W_{0}^{n+1}=\varnothing$ is obvious from the definition of $W_{0}^{n+1}=S_{0}^{n+1}$, and also the other three conditions are obviously satisfied.

Lett us assume that $U_{\mu}^{i}$ are defined for $\beta<\alpha$; then putting

$$
V_{u}^{i}=\bigcup_{1<i k} U_{\beta}^{i} \text { and } \quad U_{u}^{i}=U_{u}-\bar{V}_{u}^{i} \cup \bar{W}_{u}^{n-i+2} \quad(i=1, \ldots, n+1)
$$

Wo grot $U_{n}^{t}$ satikfying 1)-4). Since the validity of 1), 2), 4) for $U_{u}^{i}$ is (loar from the above definition, we prove 3 ) only. If $M_{\in} \in \mathfrak{X}_{m+1}$ is an an'bitanry soti containod in $U_{a}$, then

$$
M_{\sim} \cdots W_{a}^{n+1} \subseteq U_{u} \cap W_{a}^{n+1}=U_{a} \cap S_{u}^{n+1} .
$$

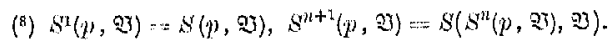


On the other hand $U_{a} \cap S_{a}^{n+1}=\varnothing$ is easily seen from the fact that svery $S^{n+2}\left(p, \mathfrak{U}_{m+1}\right)$ intersects at most $n+1$ sets of $U_{\gamma}(\gamma \geqslant \alpha)$. For let $x \in U_{a} \cap S^{n+1}$; then $S^{n+1}\left(x, 2 x_{m+1}\right)$ intersects $U_{a}$ and some $n+1$ sets of $U_{\gamma}(\gamma=\alpha)$, which is impossible. In consequence we, get $M \cap W_{a}^{n \cdot 1}=\emptyset$. Irence it follows that either $M \cap W_{a}^{i}=\emptyset(i=1, \ldots, n+1)$ or $M \cap W_{a}^{n \cdots i+2} \neq \emptyset, M \cap W_{a}^{n-i+1}$ $=\varnothing$ for some $i$ such that $2 \leqslant n-i+3 \leqslant n+1$.

If the former is the case, then $M \cap W_{a}^{1}=\emptyset$. Since $U_{a} \subset S_{\beta}^{1} \subset W_{\beta}^{1}$ is obvious for every $\beta<\alpha$, and since $U_{\beta}^{n+1} \cap W_{\beta}^{1}=\varnothing(\beta<\alpha)$ from the assumption of induction, it follows that $U_{\alpha} \cap U_{\beta}^{n+1}=\emptyset$ for every $\beta<a$. Therefore $U_{a} \cap V_{a}^{n+1}=\emptyset$, which implies $M \cap V_{a}^{n+1}=\emptyset$. Oombining this with $M \cap W_{a}^{1}=\varnothing$ we conclnde that $M \subset O_{a t}^{n+1}$.

If the latter is the case, i.e.,

$$
y \in M \cap W_{a}^{n-i+2} \neq \varnothing, \quad M \cap W_{a}^{n+i+8}=\varnothing, \quad 2, \cdots \cdots+i+30 \cdots+1,
$$

then $y \in S_{a}^{n-i+2+k}$ for some $k>0$, i.e. $S^{n-i+2+k}\left(y, \mathfrak{U}_{m+1}\right)$ intersects some $n-i+2+k$ sets of $U_{y}(\gamma>\alpha)$. Accordingly $S^{n-i+2+2+k+1}\left(x, \mathfrak{H}_{m+1}\right)$ intersects $n-i+2+k+1$ sets of $U_{y}(\gamma>a)$ for every $x \in M$. Honce

$$
x \in S_{\beta}^{n-i+s+k} \subseteq W_{\beta}^{n-i+8} \quad \text { for every } \beta<a,
$$

and hence $M \subseteq W_{\beta}^{n-i+8}$. Since $U_{\beta}^{i-1} \cap W_{\beta}^{n-i+1-8}=\varnothing(\beta<\alpha)$ from the assumption of induction, we get $M \cap U_{\beta}^{i-1}=\emptyset(\beta<\alpha)$ and consequently $M_{\cap} V_{-i 1}^{i-1}=\emptyset$. Combining this conchusion with the assumption $M_{n}$ $\cap W_{n}^{n-i+8}=\emptyset$ we obtain

$$
M \cap\left(\bar{V}_{a}^{i-1} \cup \bar{W}_{a}^{n-i-1 ~}\right)=: \emptyset
$$

from the openness of $M$. Therefore $M \subseteq U_{a}^{i-1}$. Thns the condition 3 ) is valid for $a$, and hence we can define $U_{a}^{i}(i=1, \ldots, n+1)$ satjsfying 1$\left.)-3\right)$ for every $a<\tau$.

2. Since

if we put

$$
\mathfrak{W}_{n+2}^{*}<\mathfrak{U}_{m+1}<\left\{U_{a}^{i} \mid i=1, \ldots, n+1 ; a<\tau\right\}
$$

$$
\left.\mathfrak{W}_{m}^{i}=\left\{U_{u}^{i}-\overline{S\left(R-U_{a}^{i}\right.}, \mathfrak{u}_{m+2}\right) \mid \alpha \prec \tau\right\}
$$

then $\bigcup_{i=1}^{n+1} \mathfrak{r}_{m}^{i}$ is an open covering refining $\mathfrak{u}_{m}$, and $U_{1}, U_{2} \in \mathfrak{l}_{m}^{i}$ and $U_{1} ; U_{2}$ imply

$$
S\left(U_{1}, \mathfrak{U}_{m+2}\right) \neg S\left(U_{2}, \dot{\mathfrak{u}}_{m+2}\right)=\varnothing
$$

by condition 2) of $\mathfrak{r}_{m}^{i}$. From now on let us denote $\mathfrak{H}_{2 m-1}$ and $\mathfrak{L f}_{2 m m-1}^{i}$ by $\mathfrak{H}_{m}$ and $\mathfrak{U}_{m}^{i}(m=1,2, \ldots)$ respectively for brevity; then $\mathfrak{U}_{m}$ and $\mathfrak{l}_{m}^{i}$ satisfy
(B) $\quad \mathfrak{U}_{m+1}<\bigcup_{i=1}^{n+1} \mathfrak{U}_{m}^{i}<\mathfrak{U}_{m}$,

(B') $S\left(U_{1}, \mathfrak{U}_{m+1}\right) \cap S\left(U_{2}, \mathfrak{U}_{m+1}\right)=\emptyset$

if $U_{1}, U_{2} \in \mathfrak{U}_{m}^{i}, U_{1} \neq U_{2}$.

For a fixed $i$ and for every $U \in \mathfrak{U}_{2 k-1}^{i}$ we define inductively

$\mathbb{S}(U): \mathfrak{S}^{1}(U)=\left\{U^{\prime} \mid U^{\prime} \in \mathfrak{U}_{2 k-1+2 j}^{l}\right.$ for some positive integer $j$,

$$
S\left(U^{\prime}, \mathfrak{H}_{: k-1+2 j}\right) \cap U \neq \emptyset_{\xi},
$$

$$
\mathfrak{S}^{m+1}(U)=\bigcup\left\{\subseteq\left(U^{\prime}\right) \mid U^{\prime} \in \mathfrak{S}^{m}(U)\right\} \quad(m=1,2, \ldots) .
$$

(O) From now on we denote by $U \propto U^{\prime}$ the fact that

$$
S\left(U^{\prime}, \mathfrak{U}_{2 k-1 \mid 2 j}\right) \cap U \neq \varnothing \quad \text { for } \quad U^{\prime} \in \mathfrak{X}_{2 k-1+2 j}^{i}, \quad U \in \mathfrak{U}_{2 k-1}^{i}
$$

Then

$$
\begin{aligned}
& \Xi^{m}(U)=\left\{U^{\prime} \mid U \leftarrow U_{1} \leftarrow U_{2} \leftarrow \ldots<U_{m}=U^{\prime} \text { for } U_{j} \in \mathfrak{U}_{2 k-1+n(j)}^{i}\right. \\
&(j=1,2, \ldots), 0<n(1)<n(2)<\ldots<n(m)\} .
\end{aligned}
$$

Furthermore, we define

$$
S(U)=U \cup\left\{U^{\prime} \mid U^{\prime} \epsilon \bigcup_{m=1}^{\infty} \mathbb{S}^{m}(U)\right\}
$$

The principal object of the second part is to prove that

(i) $U_{1}, U_{2} \in \mathfrak{U}_{2 k-1}^{i}$ and $U_{1} \neq U_{2}$ imply $S\left(U_{1}\right) \cap S\left(U_{2}\right)=\emptyset$,

(ii) $U_{1} \in \mathfrak{H}_{2 k-1}^{i}$ and $U_{2} \in \mathfrak{U}_{2 k-1+l}^{i}$ for some eren $l \geqslant 2$ imply $S\left(U_{2}\right) \subset S\left(U_{1}\right)$ or $S\left(U_{1}\right) \cap S\left(U_{\mathrm{a}}\right)=\emptyset$.

To prove (i) we take an arbitrary $V \in \bigcup_{m=1}^{\infty} S^{m}\left(U_{1}\right)$. If $V \in \mathbb{S}^{j}\left(U_{1}\right)$, then there exists a sequence

$U_{1}=V_{0} \leftarrow V_{1} \leftarrow Y_{2} \leftarrow \ldots<-V_{j}=r \quad$ of $\quad V_{p} \in \mathfrak{H}_{2 k-1+n(p)}^{i} \quad(p=0,1, \ldots, j)$

for some evon numbers $n(p)(p=0,1, \ldots, j)$ such that $n(p+1) \geq n(p)+2$. Especially we notice that $n(1) \geqslant n(0)+2=2$. Since $\mathfrak{U}_{2 k-1+n(p)}^{*}<\mathfrak{U}_{2 k-1+n(p)-1}$ and $\mathfrak{H}_{m}^{i}<\mathfrak{U}_{m}$ by $(\mathrm{B})$, from (C) combined with the above remark we easily see that

$$
\begin{aligned}
& V_{j} \subseteq S\left(V_{j}, \mathfrak{U}_{2 k-1 \mid n(j)}\right) \subseteq S\left(V_{j-1}, \mathfrak{U}_{2 k-1+n(j-1)}\right) \subseteq S\left(V_{j-2}, \mathfrak{U}_{2 k-1+n(j-2)}\right) \\
& C \ldots \operatorname{CS}\left(V_{1}, \mathfrak{U}_{2 l l-1+n(1)}\right) \subset U^{\prime} \text { for some } U^{\prime} \in \mathfrak{U}_{2 k-1+1} \text {. }
\end{aligned}
$$

Since

$$
U^{\prime} \cap U_{1} \supset S\left(V_{1}, U_{j t-1+n(1)} \cap U_{1} \neq 0 \text { by the fact that } U_{1}+-V_{1}\right.
$$


we obtain

(I) $V \subseteq U^{\prime}$ for every $V \in \bigcup_{m=1}^{\infty} S^{m}\left(U_{1}\right), \quad U_{1} \in U_{m k 1}^{i}$ thend for somo $U^{\prime} \in \mathfrak{U}_{2 / 3-1+1}$ with $U^{\prime} \cap U_{1} \neq \emptyset$.

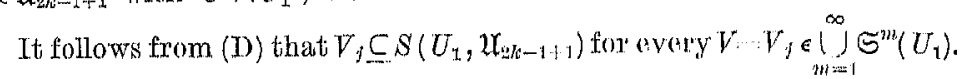
Therefore we can conclude that:

(U) $S\left(U_{1}\right) \subset S\left(U_{1}, \mathfrak{H}_{2 \gamma_{c-1+1}}\right)$ for overy $U_{1} \in \mathfrak{U}_{\Psi_{i-1-1}}$.

In consequence, if $U_{1}, U_{2} \in \mathfrak{L}_{2 t}^{l}$ and $U_{1} \neq U_{2}$, thon $b_{1} y\left(B^{\prime}\right)$ wa can

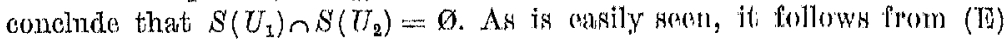
that

(F) $\left\{S(U) \mid U \in \mathfrak{X}_{\mathfrak{W} / k-1}^{i}\right\}<\mathfrak{U}_{2 k \cdots 1}^{*}$

which will be usod later.

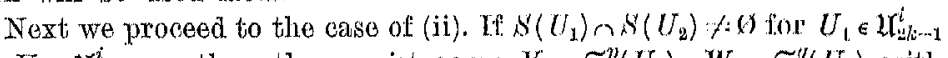
and $U_{2} \in \mathfrak{U}_{2, t-1+t}^{i}$, then there exist some $V_{p} \in \mathfrak{S}^{p}\left(U_{y}\right), W_{q} \in \mathfrak{S}^{\prime \prime}\left(U_{2}\right)$ witil $r_{p} \cap W_{q} \neq \varnothing$ and consequentily two soquencess

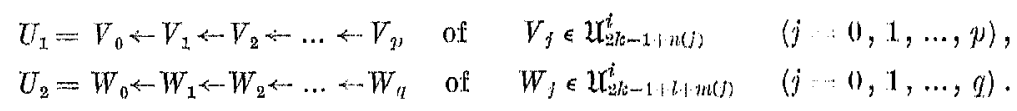

Wo take $j>0$ such that

we notice that

$$
2 k-1+n(j)=2 k-1+l<2 k-1+m(j+1)
$$

(G) $2 k-1+l+2-2 k-1+n(j+1)$,

because $l$ and $n(j+1)$ anc even. Since $2 k-1+n(j) \cdots 2 k \cdots+1 \cdot l$ imjolios $S\left(U_{2}\right)=S\left(V_{j}\right) \subseteq S\left(U_{1}\right)$ by $(\mathrm{i})$, we assume

(H) $2 k-1+n(j)<2 k-1+7$.

If $j=p$, i.e. $2 k-1+n(p)<2 k \cdots 1+1$, then since by (I) theres exists $W^{\prime} \in \mathfrak{U}_{2 k-1+l+1}$ such that $W^{\prime} \supset W_{q}$ and $W^{\prime} \cap U_{2} \neq A$, wo got

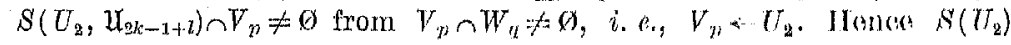
$\subseteq S\left(\nabla_{p}\right) \subseteq S\left(U_{1}\right)$

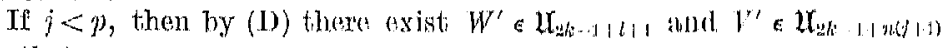
stuch that

(I) $W^{\prime} \supset W_{a}, \quad W^{\prime} \cap U_{2} \neq 0, \quad V^{\prime} \supset V_{2}, \quad V^{\prime} \cap V_{111} \neq 0$.

(Tf $j+1=p$, then. wo put $V^{\prime}:: V_{n}$.)

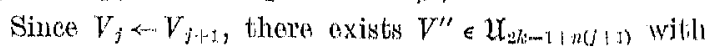

(J) $V^{\prime \prime} \cap V_{j+1}^{r} \neq \emptyset, \quad V^{\prime \prime} \cap V_{j} \neq \emptyset$.

Therefore we get

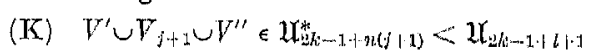
from (G). Since $V^{\prime} \cap W^{\prime} \supseteq V^{\prime} \cap W_{q} \neq 0$ from (I), it follows from (I), (J)
and (K) that

and

$$
W^{\prime} \cup V^{\prime} \cup V_{j+1} \cup V^{\prime \prime}=W^{\prime \prime} \in \mathfrak{U}_{2 k-1+l+1}^{*}<\mathfrak{U}_{2 k-1+l}
$$

$$
W^{\prime \prime} \cap U_{2} \supseteq W^{\prime} \cap U_{2} \neq \emptyset, \quad W^{\prime \prime} \cap V_{j} \supseteq V^{\prime \prime} \cap V_{j} \neq \varnothing \text {. }
$$

Thus we deduce $V_{j} \leftarrow U_{2}$ from (H) and consequently $S\left(U_{2}\right) \subseteq S\left(V_{3}\right) \subseteq S\left(U_{1}\right)$.

3. We put $\mathfrak{S}_{m}^{i}=\left\{S(U) \mid U \in \mathfrak{U}_{a_{m-1}}^{i}\right\}$ and define inductively open collections ${ }_{m} \mathfrak{S}_{m+j}(j=1,2, \ldots)$ by

${ }_{m} \mathfrak{S}_{m+1}^{i}=\mathfrak{S}_{m}^{i} \cup\left\{S \mid S \in \mathbb{S}_{m+1}^{i}, S_{\neq}^{\ddagger} S^{\prime} \quad\right.$ for every $\left.S^{\prime} \in \mathbb{S}_{m}^{i}\right\}$,

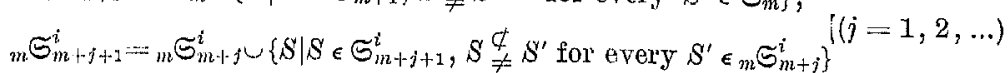
for a fixed $m$. Then $\mathfrak{I}_{m}^{i}=\bigcup_{j=1}^{\infty}{ }_{m} \widetilde{S}_{m+j}^{i}$ is a disjointed collection from (i), (ii) of 2. It follows from

$$
m+1 \mathbb{S}_{m+1+j}^{i}<\bigcup_{k=0}^{j} \mathfrak{S}_{m+1+k}^{i}<. m \Im_{m+1+j}^{i}
$$

that

$$
\text { (L) } \quad \mathfrak{I}_{m+1}^{i}=\bigcup_{j=1}^{\infty} m+1, \mathfrak{S}_{m+1+j}^{i}<\bigcup_{j=2}^{\infty} \mathfrak{S}_{m+j}^{i}<\bigcup_{j=1}^{\infty} \mathfrak{S}_{m+j}^{i}=\mathfrak{I}_{m}^{i}
$$

Since

$$
\bigcup_{i=1}^{n+1} \Xi_{m}^{i}>\bigcup_{i=1}^{n+1} \mathfrak{U}_{2 m-1}^{i}>\mathfrak{U}_{2 m}
$$

by (B), $\bigcup_{i=1}^{n+1} \Im_{m}^{i}$ is an open covering of $R$; moreover it is a refinement of $\mathcal{U}_{2 m-1}^{*}$ by $(F)$. In consequence $\left\{S\left(p, \bigcup_{i=1}^{n+1} \Theta_{m}^{i}\right)_{\mid} m=1,2, \ldots\right\}$ is a nbd basis for evory point $p$ of $R$; henco it follows from $\mathfrak{S}_{m}^{i} \subseteq \mathfrak{I}_{m}^{i}$ that $\left\{\mathfrak{I}_{m}^{i} \mid i=1, \ldots\right.$, $n+1 ; m=1,2, \ldots\}$ is an open basis of $R$. Combining this conclusion with (L), wo get $n+1$ sequences $\mathfrak{I}_{1}^{i}>\mathfrak{I}_{2}^{i}>\ldots(i=1, \ldots, n+1)$ of disjointed eollections such that $\left\{\mathfrak{I}_{m}^{i}\right\}$ is an open basis of $R$. Thus we conclude that $\operatorname{dim} R \cdots n$ by Theorem 1 .

From this thoorem we casily obtain the following main theorem.

'Thuming 3.' In order that a $T_{1}$-topological space $R$ be a metrizable space with dim $R \cdot n$ it is necessary and sufficient that there exists a sequenee $\mathfrak{B}_{1}>\mathfrak{B}_{2}^{*} \ldots \mathfrak{B}_{2}>\mathfrak{B}_{3}^{*}>\ldots$ of open coverings such that $\left\{S\left(p, \mathfrak{B}_{m}\right)\right\}$ $m=1,2, \ldots\}$ is a nud basis for each point $p$ of $R$ and such that order $\mathfrak{B}_{m}, n+1 \quad(m=1,2, \ldots)$.

Fundamental Mathemattcae, T. XLV. 
Proof. Since the necessity is contained in Theoren 2, wo prove. only the sufficiency. Iet $\mathfrak{B}_{1}=\left\{V_{\alpha} \mid \alpha \in A\right\}$; then we define $U_{n}$ by

(A) $U_{a}=\bigcup\left\{\boldsymbol{V} \mid S\left(\boldsymbol{V}, \mathfrak{B}_{2}\right) \subseteq V_{a}, V \in \mathfrak{B}_{2}\right\}$.

Since $\mathfrak{V}_{2}^{*}<\mathfrak{V}_{1}, \mathfrak{H}_{1}=\left\{U_{a} \mid \alpha \in A\right\}$ is an open covering of $R$ such that $\mathfrak{H}_{2}>\mathfrak{P}_{2}$. Furthermore we notice that

$\left(B_{1}\right)$ each set of $\mathfrak{W}_{2}$ intersects at most $n+1$ sets of: $\mathfrak{X}_{1}$ by $(A)$ and. the condition: order $\mathfrak{B}_{m} \leqslant n+1$.

Next, we assume $\mathfrak{B}_{\mathbf{3}}=\left\{V_{\theta} \mid \beta \in B\right\}$ and define $V_{\beta}$ by

$$
U_{\beta}=\bigcup\left\{V \mid S\left(\boldsymbol{V}, \mathfrak{B}_{4}\right) \subseteq V_{\beta}, V \in \mathfrak{B}_{4}\right\} \text {. }
$$

Then $\mathfrak{U}_{3}=\left\{U_{\beta} \mid \beta \in B\right\}$ is an open covering of $R$, and it follows finom $\mathfrak{H}_{3}<\mathfrak{B}_{3}$ that

We notice that

$$
\mathfrak{U}_{1}>\mathfrak{B}_{2}>\mathfrak{B}_{3}^{*}>\mathfrak{U}_{3}^{*}>\mathfrak{H}_{3}>\mathfrak{W}_{4}
$$

$\left(B_{2}\right)$ each set of $\mathfrak{B}_{4}$ intersects at most $n+1$ sets of $\mathfrak{H}_{3}$.

Thus we can repeat this process and get a sequence

$$
\mathfrak{U}_{1}>\mathfrak{B}_{2}>\mathfrak{U}_{8}^{*}>\mathfrak{U}_{3}>\mathfrak{B}_{4}>\mathfrak{U}_{6}^{*}>\mathfrak{U}_{5}>\mathfrak{B}_{43}>\ldots
$$

of open coverings such that

$\left(\mathbf{B}_{m}\right)$ each set of $\mathfrak{B}_{2 m}$ intersects at most $n+1$. sets of $\mathfrak{H}_{2 m-1}$.

Hence by $\left(\mathfrak{B}_{m}\right) \mathfrak{U}_{1}>\mathfrak{U}_{3}^{*}>\mathfrak{U}_{3}>\mathfrak{U}_{5}^{*}>\ldots$ is a sequence such that each set of $\mathfrak{U}_{2 m+1}$ intersects at most $n+1$ sets of $\mathfrak{U}_{2 m-1}$ and such that $\left\{\boldsymbol{S}\left(p, \mathfrak{U}_{2 m-1}\right)\right.$ $m=1,2, \ldots\}$ is a $n b d$ basis of $p$. Therefore we conclude that $\operatorname{dim} R, \cdots$ from Theorem 2.

First, let us apply our theorem to the notion "length of a multiplicative covering" due to Alexandroff and Kolmogoroff (see [1]).

Definition. We call a covering $\mathfrak{U}$ a multiplicative covering if. every non-empty intersection $\bigcap_{i=1}^{k} U_{i}$ of elements $U_{i}(i=1, \ldots, k)$ of $\mathfrak{U}$ is an element of $\mathfrak{U}$.

DEFINITION. The maximal number $n$ such that there exists a sequence $U_{1 \neq}^{\not p} U_{2} \neq \ldots \ldots \neq \ldots$ called the length of $\mathfrak{U}$.

Definition. We mean by the rank of an element $U$ of a multiplicative covering $\mathfrak{U}$ the maximal number $r$ such that there exists a soquence $U=U_{1} \underset{\neq}{\not} U_{2} \neq \cdots \neq . \neq p$

THEOREM 4. In order that a $T_{1}$-space $R$ be a metrizable space with $\operatorname{dim} R \leqslant n$ it is necessary and sufficient that there exists a sequence $\mathfrak{H}_{1}>\mathfrak{U}_{2}^{*}>\mathfrak{U}_{2}>\mathfrak{H}_{3}^{*}>\ldots$ of multiplieative coverings with length $\varkappa_{*} n+1$ such that $\left\{S\left(p, \mathfrak{U}_{m}\right) \mid m=1,2, \ldots\right\}$ is a nbd basis of $p$.
Proof. If $\operatorname{dim} R<n$, then there exists, by Theorem 3 , a sequence $\mathfrak{U}_{1}>\mathfrak{U}_{1}^{*}>\mathfrak{U}_{2}>\ldots$ of coverings of order $\leqslant n+1$ such that $\left\{S^{\prime}\left(p, \mathfrak{U}_{m}\right)\right.$ $m=1,2, \ldots\}$ is a nbd basis of $p$. Since $\mathfrak{U}_{m}$ plus all the intersections of a finite number of elements of $\mathfrak{H}_{m}$ is obviously a multiplicative covering with length $\leqslant n+1$, the necessity is valid.

Let us assume the existence of a sequence satisfying the condition of the proposition. If we demote by $U_{r, 1}\left(a \in A_{r}\right)$ all the elements of $\mathfrak{U}_{1}$ with rank $r$, then

$$
\mathfrak{U}_{1}=\left\{U_{r \alpha} \mid a \in A_{r}, \eta=1, \ldots, n+1\right\} .
$$

Wo define $V_{m}^{(i)}(i=1, \ldots, n+1)$ by

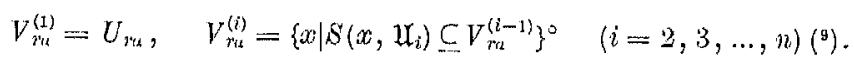

It follows directly from the above definition and $\mathfrak{W}_{i}^{*}<\mathfrak{U}_{i-1}$ that

(A) $V_{r t}^{(n+1)} \subseteq \ldots \subseteq V_{r t}^{(2)} \subseteq V_{r+1}^{(1)}=U_{r, t}$;

(B) $\mathfrak{H}_{i}<\left\{V_{r a}^{(i)} \mid a \in A_{r}, r=1, \ldots, n+1\right\} \quad(i=1, \ldots, n+1)$,

(C) $S\left(V_{r_{u}}^{(i)}, \mathfrak{U}_{i}\right) \subseteq V_{r \alpha}^{(i-1)} \quad(i=2, \ldots, n+1)$.

Next we define $M_{r_{t}}\left(\eta^{*}=1, \ldots, n+1\right)$ by

$$
\begin{aligned}
& M I_{1 u}=V_{1 u}^{(1)}=U_{1 a}
\end{aligned}
$$

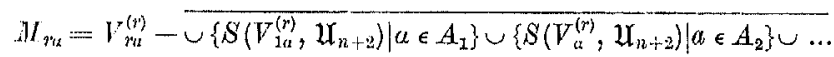

$$
\begin{aligned}
& \overline{\cup\left\{S\left(V_{r-1,1,}^{(r)}, \mathfrak{U}_{n+2}\right) \mid \alpha \in A_{r-1}\right\}} \quad(r=2, \ldots, n+1) .
\end{aligned}
$$

Iet us show that

(E) $\mathfrak{\mathfrak { U }}_{n+2}<\mathfrak{M}_{1}=\left\{M_{r_{\alpha}} \mid \alpha \in A_{*}, v^{*}=1, \ldots, n+1\right\}$.

Let $U$ be an arbitrary set of $\mathfrak{U}_{n+2}$; then by nsing (B) for $i=n+1$ we get $V_{\gamma^{\prime}}^{(n+1)}$ with $U \subset V_{r+1}^{(n+1)} . U \subseteq V_{r t}^{(r)}$ follows from $(A)$, and hence

$$
\mathfrak{U}_{n+!}<\left\{V_{r a}^{(r)} \mid \alpha \in A_{r}, \mu=1,2, \ldots, n+1\right\} .
$$

Therefole we an find for every $U \in \mathfrak{U}_{n-2}$ the minimum number $r$ such that $U \subset Y_{m}^{(r)}$. To prove (W) we show that,

$$
\operatorname{UnS}\left(V_{l u}^{(p)}, \mathfrak{U}_{n+2 \cdot 2}\right)=\varnothing
$$

for this $r$ and overy $k$ with $1 \leqslant b \leqslant \psi-1$ and for every $a \in A_{k}$. If we assume the contrary: $U \cap S\left(V_{k+1}^{(n)}, \mathfrak{U}_{n+2,2}\right) \neq 0,1 \leqslant \gamma_{k} \leqslant r-1, a \in A_{k}$, then we have, from $\mathfrak{H}_{v+2}^{*}<\mathfrak{U}_{N},(\mathrm{~A})$ and $(\mathrm{O})$,

$$
U \subseteq \mathbb{S}\left(V_{k}^{(r)}, \mathfrak{X}_{r}\right) \subseteq V_{k m}^{(j-1)} \subseteq V_{k+1}^{(k)},
$$

(9) $A^{\circ}$ denotes tho interior of $A$. 
which contradicts the character of $r$ because $k<r$. Hence we must have $\sigma_{\cap} S\left(\nabla_{k x}^{(r)}, \mathfrak{U}_{n+2}\right)=\varnothing\left(1 \leqslant k \leqslant r-1, a \in A_{k}\right)$. This combined with $U \subseteq V_{r \times x}^{(r)}$ for a definite $a \in A_{r}$ implies $U \subseteq M_{r a}$ by (D), proving (E).

Now, to show that

(F) order $\mathfrak{M}_{1} \leqslant n+1$,

\section{we prove}

(G) $M_{r_{\alpha}} \cap M M_{r_{\beta}}=\emptyset$ for $\alpha \neq \beta$.

In the case $\alpha, \beta \in A_{1}, \alpha \neq \beta$ implies clearly

$$
M M_{1 n} \cap M_{1 \beta}=U_{1 n} \cap U_{1 ;}=\Phi,
$$

because the ranks of $U_{1 \alpha}$ and of $U_{1 \beta}$ are 1 .

To show the same assertion for $r>1$, we prove that

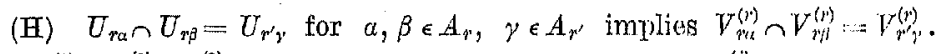
First, $\nabla_{r_{\gamma}}^{(2)} \subseteq V_{r \alpha}^{(2)} \cap V_{r \beta}^{(2)}$ is obvious from the definition of $V_{r \alpha}^{(i)}$. Oonversely, suppose that $x \in V_{r a}^{(2)} \cap V_{r \beta}^{(2)}$; then there exist nbds $P(x), Q(x)$ of $x$ such that

Hence

$$
S\left(P(x), \mathfrak{H}_{2}\right) \subseteq U_{r^{\prime \prime}}, \quad S\left(Q(x), \mathfrak{H}_{2}\right) \subseteq U_{r^{\prime \prime}} .
$$

$$
S\left(P(x) \cap Q(x), \mathfrak{H}_{2}\right) \subseteq U_{r^{\prime} \cap} \cap U_{r \beta}=U_{r^{\prime} \gamma} .
$$

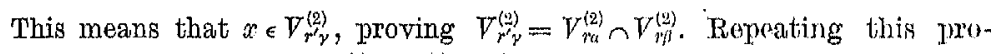
cess, we conclude that $V_{r^{\prime} y}^{(r)}=V_{r a}^{(r)} V \cap V_{\gamma \beta}^{(r)}$.

We now return to the proof of $(\mathrm{G})$. By using $(\mathrm{H})$ and $(\mathrm{D})$, wo have

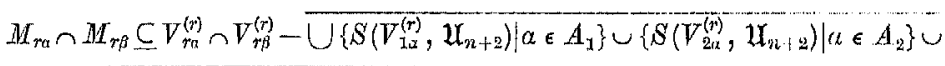

$$
\begin{aligned}
& \ldots \cup\left\{S\left(V_{r-1, \alpha}^{(r)}, \mathfrak{U}_{n+2}\right) \mid \alpha \in A_{r-1}\right\} \subseteq V_{r \alpha}^{(r)} \cap V_{r \beta}^{(r)}-S\left(V_{r \mu}^{(r)}, \mathfrak{U}_{n+2}\right)=\emptyset
\end{aligned}
$$

for $r^{\prime}$ determined by $U_{r a} \cap U_{r \beta}=U_{r^{\prime} \gamma}$, because $r^{\prime}<r$ and consequently

$$
S\left(\nabla_{r \gamma}^{(r)}, \mathfrak{U}_{n+2}\right) \subseteq \cup\left\{S\left(V_{1 a}^{(r)}, \mathfrak{U}_{n+2}^{\gamma}\right) \mid \alpha \in A_{1}\right\} \cup \ldots \cup\left\{S\left(V_{r-1, a}^{(r)}, \mathfrak{U}_{n-1}\right) \mid \alpha \in A_{r-1}\right\} .
$$

Thus $(\mathrm{G})$ is proved for $r=1, \ldots, n+1$. Since $\mathfrak{M}_{1}=\left\{M_{r \alpha} \mid \alpha \in A_{r}, r=1, \ldots\right.$, $n+1\}$, the assertion $(\mathrm{F})$ : order $\mathfrak{M}_{1} \leqslant n+1$ follows directly from $(\mathrm{K})$.

Since $\mathfrak{M}_{1}<\mathfrak{U}_{1}$ is obvious," from (D) combined with (Vi) wo obtain a covering $\mathfrak{M}_{1}$ satisfying

$$
\mathfrak{U}_{n+2}<\mathfrak{M}_{1}<\mathfrak{U}_{1}, \quad \text { order } \mathfrak{M}_{1} \leq n+1
$$

Repeating the same process, we get a sequence $\mathfrak{M}_{m}(m, \ldots, 1,2, \ldots)$ of coverings of order $\leqslant n+1$ such that

$$
\mathfrak{U}_{1+m(n+1)}<\mathfrak{M}_{m}<\mathfrak{H}_{1+\{m-1)(n+1)} .
$$

Therefore we have, from Theorem $3, \operatorname{dim} R<n$.
\$ 2. Dimension and metric function. We know that if $\varrho\left(x, y_{i}\right)<\varepsilon\left({ }^{10}\right)(i=1,2,3)$ in Euclidean 1 -space $E_{1}$, then $\varrho\left(y_{i}, y_{j}\right)<\varepsilon$ for some two points $y_{i}, y_{j}$ of the three points $y_{1}, y_{2}, y_{3}$ and the same is also valid for seven points $y_{i}(i=1, \ldots, 7)$ and a point $x$ of $E_{2}$, and that the number of $y_{i}$ having such character increases with the dimension 17 of $E_{n}$. To begin with, we shall characterize generally the dimension of a metric space with a similar property of metric function.

THEorem 5. In order that $\operatorname{dim} R \leqslant n$ for a metrizable space $R$ it is necessary and sufficient to be able to define a metric $\varrho(x, y)$ agreeing with the topology of $R$ such that for every $\varepsilon>0$ and for every point $x$ of $R$,

imply

$$
\varrho\left(S_{\varepsilon / 2}(x), y_{i}\right\rangle<\varepsilon \quad(i=1, \ldots, n+2)
$$

$$
\varrho\left(y_{i}, y_{j}\right)<\varepsilon \text { for some } i, j \text { with } i \neq j \text {. }
$$

Proof. Necessity. 1. Let $R$ be a metrizable space with $\operatorname{dim} R \leqslant n$; then by Theorem 2 there exists a sequence $\mathfrak{U}_{1}>\mathfrak{U}_{2}^{* *}>\mathfrak{U}_{2}>\mathfrak{U}_{3}^{* * *}>\ldots$ $\left(\mathfrak{U}^{* *}=\left(\mathfrak{I}^{*}\right)^{*}\right)$ of open coverings of $R$ such that $\left\{S\left(p, \mathfrak{U}_{m}\right) \mid m=1,2, \ldots\right\}$ is a nbd basis for each point $p$ of $R$ and such that each $S^{2}\left(p, \mathfrak{H}_{m+1}^{*}\right)$ intersects at most $n+1$. sets of $\mathfrak{H}_{m}$. Now we define $S_{m_{2}, m_{3}, \ldots, m_{p}}(U)$ for $1 \leqslant m_{1}$ $<m_{2}<\ldots<m_{p}$ and for $\sigma \in \mathfrak{U}_{m_{1}}$ by

$$
\begin{aligned}
S_{m_{2}}(U) & =\bigcup\left\{U^{\prime} \mid S\left(U^{\prime}, \mathfrak{U}_{m_{2}}\right) \cap U \neq \emptyset, U^{\prime} \in \mathfrak{U}_{m_{2}}\right\}=S^{2}\left(U, \mathfrak{U}_{m_{2}}\right), \\
S_{m_{3}, \ldots, m_{p}}(U) & =\bigcup\left\{U^{\prime} \mid S\left(U^{\prime}, \mathfrak{U}_{m_{p}}\right) \cap S_{m_{2}, \ldots, m_{p-1}}(U) \neq \emptyset, U^{\prime} \in \mathfrak{U}_{m_{p}}\right\} \\
& =S^{2}\left(S_{m_{2,}, \ldots, m_{p-1}}(U), \mathfrak{U}_{m_{p}}\right)
\end{aligned}
$$

and

$$
S_{m_{2}, \ldots, m_{p}} \cdot(U)=U \text { for } \quad p=1 .
$$

Furthermore we define open coverings of $R$ by

$$
\mathfrak{S}_{m_{\mathrm{I}}}=\mathfrak{H}_{m_{1}}, \quad \mathbb{S}_{m_{1}, \ldots, m_{p}}=\left\{S_{m_{2}, \ldots, m_{p}}(U) \mid U \epsilon \mathfrak{U}_{m_{1}}\right\} .
$$

We show first that

(A) $\frac{1}{2^{m_{1}}}+\ldots+\frac{1}{2^{m_{p}}} \geqslant \frac{1}{2^{l_{1}}}+\ldots+\frac{1}{2^{l_{q}}}$ implies $\quad \Im_{m_{1}, \ldots, m_{p}}>\Im_{l_{1}, \ldots, l_{q}}$.

Since in the case $p \geqslant q$ and $m_{i}=l i(i=1, \ldots, q)$ the validity of $(\mathrm{A})$ is evident from the definition, we concern ourselves with the other cases only. We can easily prove the important proposition:

(B) $\mathfrak{S}_{k_{2}, \ldots, k_{y}}\left(U^{\prime}\right) \subseteq S^{\prime}\left(U^{\prime}, \mathfrak{U}_{k_{k_{1}}}\right)$ for every $U^{\prime} \in \mathfrak{U}_{k_{1}}$,

${ }^{100)}$ We denote by $\varrho(x, y)$ the distance of $x$ and $y$; see $\left({ }^{5}\right)$. 
which will often be used in the remainder of this proof. For it follows from $k_{1}<k_{2}<\ldots<k_{r}$ that

$$
\mathfrak{U}_{k_{1}}>\mathfrak{U}_{k_{2}}^{* *}>\mathfrak{U}_{k_{3}}>\mathfrak{U}_{k_{k_{3}}}^{* *}>\ldots>\mathfrak{l}_{k_{r}}^{* *}
$$

and hence

$$
S_{k_{2}, \ldots, k_{r}}\left(U^{\prime}\right) \subseteq S^{2}\left(S_{k_{2}, \ldots, k_{r-1}}\left(U^{\prime}\right), \mathfrak{H}_{k_{r}}\right) \subseteq S\left(S_{k_{2}, \ldots, k_{r-9}}\left(U^{\prime}\right), \mathfrak{U}_{k_{r-1}}\right)
$$

Therefore, if

$$
\subseteq \ldots \subseteq S\left(S_{k_{2}}\left(U^{\prime}\right), \mathfrak{U}_{k_{2}}\right) \subset S\left(U^{\prime}, \mathfrak{U}_{k_{3}}\right) \text {. }
$$

$$
\frac{1}{2^{m_{1}}}+\ldots+\frac{1}{2^{m_{p}}}>\frac{1}{2^{l_{1}}}+\ldots+\frac{1}{2^{i_{i}}}
$$

and

$m_{1}=l_{1}, m_{2}=l_{2}, \ldots, m_{i-1}=l_{i-1}, m_{i}<l_{i}$ for a definite $i$ with $2, i, q, q$,

then from (B) we have

(C) $S_{l_{t+1}, \ldots, l_{q}}\left(U^{\prime}\right) \subseteq S\left(U^{\prime}, \mathfrak{U}_{l_{l}}\right) \subseteq U^{\prime \prime}$ for every $U^{\prime} \in \mathfrak{U}_{l_{i}}$ and for some $U^{\prime \prime} \in \mathfrak{H}_{m_{l}}$. If further this $U^{\prime}$ satisfies $S_{l_{3}, \ldots, l_{k-1}}(U) \leftarrow U^{\prime}$ for $U \in \mathfrak{U}_{l_{1}}\left({ }^{11}\right)$, then from (C) we have

(D) $\quad U^{\prime \prime} \cap S_{m_{2}, \ldots, m_{i-1}}(U)=U^{\prime \prime} \cap S_{L_{2}, \ldots, l_{i-1}}(U) \neq \varnothing$.

Therefore by (C) and (D)

(E) $S_{L_{t+1}, \ldots, l_{g}}\left(U^{\prime}\right) \subseteq U^{\prime \prime} \subseteq S_{m_{2}, \ldots, m_{4}}(U) \subseteq S_{m_{2}, \ldots, m_{p}}(U)$ holds for overy $U^{\prime}$ with $\mathbb{S}_{l_{3}, \ldots, l_{-1}}(U) \leftarrow U^{\prime} \in \mathfrak{U}_{l_{l}}$. Hence it follows from (E) that

$S_{l_{2}, \ldots, l_{q}}(U)=S_{l_{2, \ldots, l_{l-1}}}(U) \cup\left[\cup\left\{S_{l_{i+1}, \ldots, l_{q}}\left(U^{\prime}\right) \mid S_{l_{g}, \ldots, l_{l-1}}(U) \leftarrow U^{\prime} \in \mathfrak{U}_{l_{l}}\right\}\right] \subset S_{m_{1}, \ldots, m_{y}}(U)$

proving $\Im_{l_{1}, \ldots, l_{p}}<\mathfrak{S}_{m_{1}, \ldots, m_{p}}$.

In the case of $m_{1}<l_{1}$

$$
S_{l_{2}, \ldots, l_{q}}\left(U^{\prime}\right) \subseteq S\left(U^{\prime}, \mathfrak{U}_{l_{1}}\right) \subseteq \dot{U}^{\prime \prime} \subseteq S_{9 n_{2}, \ldots, m_{p}}\left(U^{\prime \prime}\right)
$$

for every $U^{\prime} \in \mathfrak{U}_{l_{1}}$ and for some $U^{\prime \prime} \in \mathfrak{U}_{m_{1}}$ follows directly from (O). 'Lhis completes the proof of proposition (A).

2. Now we define a non-negatively valned function $\varrho(x, y)$ on $R \times R$ by

(F) $\varrho(x, y)=\inf \left\{1 / 2^{m_{1}}+\ldots+1 / 2^{m_{n}} \mid y \in S\left(x, \mathcal{S}_{m_{1}, \ldots, m_{p}}\right)\right\}$,

$\varrho(x, y)=1 \quad$ if $\quad y \notin S\left(x, \Xi_{m_{1}, \ldots, m_{p}}\right)$ for every $\quad m_{i} \quad(i=1, \ldots, p)$.

Tet us show that $\varrho(x, y)$ satisfies the axiom of metric function.

(11) We use the notation $A \leftarrow U^{\prime}$ in a somewhat different sense from that of the groof of Theorem 2, i. e., $A \leftarrow U^{\prime}$ for $U^{\prime} \in \mathfrak{U}_{l}$ means $S\left(U^{\prime}, \mathfrak{U}_{l}\right) \cap A \neq \emptyset$ in this proot.
Since $\left\{\mathcal{S}\left(p, \mathfrak{U}_{m}\right) \mid m=1,2, \ldots\right\}$ is a $\mathrm{nbd}$ basis of $p, \varrho(x, y)$ obviously agrees with the topology of $R, i . e .,\left\{S_{\varepsilon}(x) \mid \varepsilon>0\right\}$ is a nbd basis of each point $p$ of $R$.

To prove the triangle axiom $\varrho(x, y)^{\circ}+\varrho(y, z) \geqslant \varrho(x, z)$ we assume that $\varrho(x, y)=a \geqslant b=\varrho(y, z)$. For an arbitrary $\varepsilon>0$ we can select $m_{1}, \ldots, m_{p} ; l_{1}, \ldots, l_{q}$ such that

$$
\begin{array}{cl}
1 \leqslant m_{1}<\ldots<m_{p}, & 1 \leqslant l_{1}<\ldots<l_{q}, \\
a+\varepsilon>1 / 2^{m_{1}}+\ldots+1 / 2^{m_{p}}>a, & b+\varepsilon>1 / 2^{l_{1}}+\ldots+1 / 2^{l_{q}}>b
\end{array}
$$

and such that

$$
1 / 2^{m_{1}}+\ldots+1 / 2^{m} m>1 / 2^{l_{1}}+\ldots+1 / 2^{l_{0}} .
$$

Since $y \in S\left(x, \mathcal{S}_{n_{1}, \ldots, m_{q}}\right), z \in S\left(y, \mathfrak{S}_{l_{1}, \ldots, l_{q}}\right)$ are obvious from $(\mathrm{F})$, we assume

(G) $x, y \in S_{m_{2}, \ldots, m_{p}}(U), \quad U \in \mathfrak{U}_{m_{1}} ; y, z \in S_{l_{2}, \ldots, l_{p}}(V), V \in \mathfrak{U}_{l_{1}}$.

Moreover we notice that we can assume

(H) $p, q \geqslant 2, m_{p}>l_{1}$

without loss of generality.

(i) Let us consider first the case of $m_{1}=l_{1}$. Since $S_{m_{3}, \ldots, m_{p}}(U)$ $\subseteq S\left(U, \mathfrak{U}_{m_{1}}\right)$ and $S_{l_{2}, \ldots, l_{q}}(V) \subseteq S\left(V, \mathfrak{U}_{l_{1}}\right)=S\left(V, \mathfrak{U}_{m_{1}}\right)$ hold by (B), it follows from $(G)$ and $\mathfrak{U}_{m_{1}}^{* * *}<\mathfrak{H}_{m_{1}-1}$ that

$$
x, z \in S\left(U, \mathfrak{U}_{m_{1}}\right) \cup S\left(V, \mathfrak{H}_{m_{1}}\right) \subseteq W
$$

for some $W \in \mathfrak{U}_{m_{1}-1}$. Hence $z \in S\left(x, \Xi_{m_{1}-1}\right)$, which implies

$$
\varrho(x, z) \leqslant 1 / 2^{m_{1}-1} \leqslant 1 / 2^{m_{1}}+\ldots+1 / 2^{m_{p}}+1 / 2^{l_{1}}+\ldots+1 / 2^{l_{\alpha}}<a+b+2 \varepsilon
$$

because $m_{1}=l_{1}$.

(ii) To consider the case of $m_{1}<l_{1}$ we notice that there exist two sequences

$\left(I_{1}\right) \quad U=U_{1} \leftarrow U_{2} \leftarrow \ldots \leftarrow U_{p}, V=V_{1} \leftarrow V_{2} \leftarrow \ldots \leftarrow V_{q}$ with $U_{i} \in \mathfrak{U}_{m_{i}}$ $(i=1, \ldots, p), V_{j} \in \mathfrak{L}_{y_{j}}(j=1, \ldots, q), y \in U_{p} \cap V_{q}$ and such that

$\left(I_{2}\right) \quad x \in S_{m_{2}, \ldots, m_{p}}\left(U_{1}\right), \quad z \in S_{l_{2}, \ldots, l_{q}}\left(V_{1}\right)$.

By (H) wo can take $i \geqslant 1$ such that $m_{i}<l_{1} \leqslant m_{i+1}$.

a) In the case of $l_{1}<m_{i+1}$ we can select $S_{I}, S_{2} \in \mathfrak{U}_{l_{1}+1}^{*}$ snch that

$$
y \in S_{1} \cap S_{2}, \quad S_{1} \cap U_{i} \neq \varnothing, \quad S_{2} \cap V_{1} \neq \varnothing
$$

For it follows from $m_{i+1}, l_{2} \geqslant l_{1}+1$ that $V_{i+1} \in \mathfrak{U}_{m_{i+1}}<\mathfrak{U}_{l_{1}+1}$ and $V_{2} \in \mathfrak{U}_{l_{2}}$ $<\mathfrak{H}_{l_{1}+1}$. Hence

$$
y \in S_{m_{i+1}, \ldots, m_{p}}\left(U_{i+1}\right) \subseteq S\left(U_{i+1}, \mathfrak{U}_{m_{i+1}}\right) \subseteq S\left(U_{i+1}, \mathfrak{U}_{l_{1}+1}\right) \subseteq S
$$


for some $S_{1} \in \mathfrak{U}_{l_{1}+1}^{*}$ and

$$
y \in S_{g_{3}, \ldots, l_{q}}\left(\nabla_{2}\right) \subseteq S\left(V_{\dot{2}}, \mathfrak{u}_{l_{2}}\right) \subseteq S_{2}
$$

for some $S_{2} \in \mathfrak{U}_{l_{1}+1}^{*}$ follows from (B). Then $S_{1} \cap U_{i} \neq \emptyset$ and $S_{2} \cap V_{1} \neq \varnothing$ are obvious because $U_{i} \leftarrow U_{i+1}, V_{i} \leftarrow V_{2}$. On the other hand, since $y \in S_{1} \cap S_{2} \neq \varnothing$ and $\mathfrak{U}_{1_{1}+1}^{* *}<\mathfrak{U}_{l_{1}}, S_{1} \cup S_{2} \subset W$ holds for some $W \in \mathfrak{H}_{l_{1}}$. Hence $S\left(V_{1}, \mathfrak{U}_{l_{1}}\right) \cap U_{i} \neq \emptyset$, i. e., $Z_{i} \leftarrow V_{1} \in \mathfrak{U}_{l_{1}}$. We can consider $S_{l_{1}, \ldots, l_{q}}\left(U_{i}\right)$ becanse $U_{i} \in \mathfrak{U}_{m_{i}}$ and $m_{i}<l_{1}$, and hence from the above discussion we get

(J) $z \in \mathbb{S}_{l_{1}, \ldots, l_{q}}\left(U_{i}\right) \subseteq S_{m_{2}, \ldots, m_{i}, l_{1}, \ldots, l_{q}}\left(U_{1}\right)$.

By $\left(I_{2}\right)$ there exists as sequence

(K) $\quad U_{1} \leftarrow U_{2}^{\prime} \leftarrow \ldots \leftarrow U_{p}^{\prime} \ni x$ with $U_{j}^{\prime} \in \mathfrak{U}_{m_{j}}(j=2, \ldots, p)$

It follows from (B) and from $l_{1}<m_{i+1}$ that $x \in S\left(U_{i+1}^{\prime}, \mathfrak{U}_{m_{i+1}} \subseteq \subseteq S^{\prime}\right.$ for some $S^{\prime} \in \mathfrak{U}_{l_{1}}$. Since $\mathbb{S}^{\prime} \cap U_{i}^{\prime} \neq \emptyset$ by $U_{i}^{\prime} \leftarrow U_{i+1}^{\prime}$, we get $x \in \mathcal{S}_{m_{m_{2}}, \ldots, m_{1}, l_{1}}\left(U_{1}\right)$. Therefore $x, z \in S_{m_{3}, \ldots, m_{i}, l_{1}, \ldots, l_{q}}\left(U_{1}\right)$, and hence $z \in S\left(x, \mathbb{S}_{\left.m_{1}, \ldots, m_{1}, l_{1}, \ldots, l_{q}\right)}\right.$. Thus we get

$$
\varrho(x, y) \leqslant 1 / 2^{m_{1}}+\ldots+1 / 2^{m_{i}}+1 / 2^{l_{1}}+\ldots+1 / 2^{l_{a}}<a+b+2 \varepsilon .
$$

b) If $l_{1}=m_{i+1}$, then we take $k$ such that

(L) $0 \leqslant k \leqslant i ; m_{i+1}-1=m_{i}, m_{i}-1=m_{i-1}, \ldots, m_{i-k+2}-1=m_{i-k+1}$, $m_{i-k+1}-1>m_{i-k}$, where $k=0$ means $m_{i+1}-1>m_{i}$, and $k=i$ means $m_{j+1}-1=m_{j}(j=1,2, \ldots, i)$.

In the case of $k<i$ it follows from $\left(\mathrm{I}_{1}\right)$ and $(\mathrm{B})$ that $S\left(U_{i-k+1}, \mathfrak{U}_{m_{i-k+1}}\right) \cap U_{i-k} \neq \emptyset$ and $y \in \mathbb{S}\left(U_{i-k+1}, \mathfrak{U}_{m_{i-k+1}}\right) \cap S\left(V_{1}, \mathfrak{U}_{l_{1}}\right) \neq \varnothing$, which implies

(M) $W \cap U_{i-k} \neq \emptyset, W \supseteq S\left(\nabla_{1}, \mathfrak{H}_{l_{1}}\right)$

for some $W \in \mathfrak{U}_{m_{i-k+1}-1}$ because $l_{1} \geqslant m_{i-k+1}$ and $\mathfrak{U}_{m}^{* *}<\mathfrak{H}_{m-1}$. Since we can consider $S_{m_{2}, \ldots, m_{i-k}, m_{i-k+1}-1}\left(U_{1}\right)$ because of $m_{i-k_{0+1}-1}>m_{i-k}$ and since $z \in S\left(V_{1}, \mathfrak{U}_{l_{1}}\right) \subseteq W$ by $\left(\mathrm{I}_{1}\right)$ and $(\mathrm{B})$, we can conclude from $(\mathrm{MI})$ and $\left(\mathrm{I}_{1}\right)$ that

(N) $z \in \mathbb{S}_{m_{2}, \ldots, m_{i-k}, m_{i-k+1}-1}\left(U_{1}\right)$

with respect to $x$, we select a sequence satisfying (K). Then

$$
x \in S\left(U_{i-k+1}^{\prime}, \mathfrak{U}_{m_{i-k+1}}\right)_{\neq}^{\not}\left(S_{m_{2, \ldots, m_{i-k}}}\left(U_{1}\right)\right)^{c}\left({ }^{13}\right)
$$

by (B), and hence there exists $W^{\prime} \in \mathfrak{U}_{m_{i-k+1}-1}$ satisfying $x \in W^{\prime}$ $\stackrel{\Phi}{\neq}\left(S_{m_{2}, \ldots, m_{i-k}}\left(U_{1}\right)\right)^{c}$. Hence $x \in S_{m_{2}, \ldots, m_{1-k}, m_{i-k+1}-1}\left(U_{1}\right)$. This combined with (N) implies $z \in S\left(x, \Im_{m_{1}, \ldots, m_{i-k}, m_{i-k+1}-1}\right)$, and hence

$\varrho(x, z) \leqslant 1 / 2^{m_{1}}+\ldots+1 / 2^{m_{i}-k}+1 / 2^{m_{i-k+1}-1}=1 / 2^{m_{1}}+\ldots+1 / 2^{m_{i+1}}+1 / 2^{l_{1}}<a+b+2 \varepsilon$ by $l_{1}=m_{i+1}$ and $(\mathrm{L})$.

(2) $A^{c}$ denotes the complement set of $A$. Hence $B \underset{\neq}{\not \subset} A^{\circ}$ means $B \cap A \neq \emptyset$.
In the case of $k=i$ we select, by $\left(\mathrm{I}_{1}\right),\left(\mathrm{I}_{2}\right)$ and $(\mathrm{B}), P, Q \in \mathfrak{U}_{m_{1}}^{*}$ with $x \in P, z \in Q, P \cap Q \neq \emptyset$. Then $z \in S\left(x, \mathfrak{u}_{m_{1}-1}\right)\left({ }^{18}\right)$, which implies

$$
\varrho(x, z) \leqslant 1 / 2^{m_{1}-1}=1 / 2^{m_{1}}+\ldots+1 / 2^{m_{i+1}}+1 / 2^{l_{1}} \leqslant a+b+2 \varepsilon .
$$

Thus we get, in every case, $\varrho(x, z) \leqslant a+b+2 \varepsilon$ for an arbitrary $\varepsilon>0$, proving

$$
\varrho(x, z) \leqslant a+b=\varrho(x, y)+\varrho(y, z) .
$$

3. Now it remains to prove that $\varrho\left(S_{s / 2}(x), y_{i}\right)<\varepsilon(i=1, \ldots, n+2)$ imply $\varrho\left(y_{i}, y_{j}\right)<\varepsilon$ for some distinct two points $y_{i}, y_{j}$. Since $\varrho\left(S_{\varepsilon / 2}(x), y_{i}\right)<\varepsilon$, we can choose $n+2$ points $x_{i}$ and a positivo number $\delta$ such that

$$
\varrho\left(x, x_{i}\right)<\delta<\varepsilon / 2, \quad \varrho\left(x_{i}, y_{i}\right)<\varepsilon .
$$

Let $m_{1}, \ldots, m_{p}$ be positive integers satisfying

$$
2 \delta<1 / 2^{m_{1}}+\ldots+1 / 2^{m_{p}}<\varepsilon ;
$$

then there exist $S_{i} \in \mathcal{S}_{m_{1}, \ldots, m_{p}}(i=1, \ldots, n+2)$ satisfying $x_{i}, y_{i} \in S_{i}$ because of $\varrho\left(x_{i}, y_{i}\right)<\varepsilon$. On the other hand, since $\delta<1 / 2^{m_{1}+1}+\ldots+1 / 2^{m_{p}+1}$, we must have

(O) $n_{i} \in S\left(x, \mathbb{S}_{m_{1}+1_{1}, \ldots, m_{p}+1}\right)(i=1, \ldots, n+2)$

because of $\varrho\left(x_{i}, x\right)<\delta$. Let $S_{i}=S_{m_{1}, \ldots, m_{p}}\left(U_{i}\right), U_{i} \in \mathfrak{H}_{m_{1}}$; then by (B) there exists $S_{i}^{\prime} \in \mathfrak{U}_{m_{2}}^{*}$ satisfying $S_{i}^{\prime} \cap U_{i} \neq \varnothing, S_{i}^{\prime} \ni x_{i}$. Hence it follows from (0) and $\mathfrak{S}_{m_{1}+1, \ldots, m_{p}+1}<\mathfrak{H}_{m_{1}+1}^{*}$ that

which implies

$$
S_{i}^{\prime} \cap S\left(x, \mathfrak{U}_{m_{1}+1}^{*}\right) \neq \emptyset \quad(i=1, \ldots, n+2),
$$

$$
S^{2}\left(x, \mathfrak{U}_{m_{1}+1}^{*}\right) \cap U_{i} \neq \emptyset \quad(i=1, \ldots, n+2)
$$

because $\mathfrak{U}_{m_{2}}^{*}<\mathfrak{U}_{m_{1}+1}^{*}$. Since by the first assumption $\mathcal{S}^{2}\left(x, \mathfrak{U}_{m_{1}+1}^{*}\right)$ intersects at most $n+1$ sets of $\mathfrak{U}_{m_{1}}$, we must have $U_{i}=U_{j}$ for some $i, j$ with $i \neq i$. Then $y_{i} \in S\left(y_{i}, S_{m_{1}, \ldots, m_{p}}\right)$, and hence we conclude that

$$
\varrho\left(y_{i}, y_{j}\right) \leqslant 1 / 2^{m_{1}}+\ldots+1 / 2^{m_{p}}<\varepsilon
$$

Sufficieney. We denote by $\varrho(x, y)$ a metric satisfying the condition of this theorem. Then we denote by $M_{1}$ a maximal subset of $R$ such that $x, y \in M_{1}$ and $x \neq y$ imply $\varrho(x, y) \geqslant 1 / 2$. By the maximal property of $M_{1} \mathfrak{U}_{1}^{\prime}=\left\{S_{1 / 2}(\infty) \mid \infty \in M_{1}\right\}$ is evidently an open covering of $R$. Let $S_{1 / 2^{2}}(x)$ intersect each of $S_{1 / 2}\left(x_{i}\right)$ for $x_{i} \in M_{1}(i=1, \ldots, n+2)$; then it follows from the property of $\varrho(x, y)$ that $\varrho\left(x_{i}, x_{j}\right)<1 / 2$ for some distinct points

(18) Since $\varrho(x, z) \leqslant a+b+2 \varepsilon$ is obvious in the case of $m_{1}=1$, we assume $m_{1}>1$. 
$x_{i}, x_{j}$. This implies $x_{i}=x_{j}$ by the property of $M_{1}$. Therefore $S_{1 / 2}(x)$ for an arbitrary point $x$ of $R$.intersects at most $n+1$ sets of $\mathfrak{U}_{1}^{\prime}$. Put

$$
\mathfrak{U}_{1}=\left\{\bigcup\left\{S_{1 / 28}(y) \mid y \in S_{1 / 2}(x)\right\} \mid x \in M_{1}\right\}
$$

then order $\mathfrak{H}_{1} \leqslant n+1$. Using the notation $\widetilde{S}_{r}=\left\{S_{\mu}(x) \mid x \in R\right\}$, we have

$$
\mathfrak{S}_{1 / 25}^{*}<\mathfrak{S}_{1 / 2^{3}}<\mathfrak{U}_{1}<\mathbb{S}_{1 / 2+1 / 2 ;}
$$

Next we denote by $M_{2}$ a maximal subset of $R$ such that $x_{i}, y \in M I_{4}$ and $x \neq y$ imply $\varrho(x, y) \geqslant 1 / 2^{6} . \mathfrak{X}_{2}^{\prime}=\left\{S_{1 / \mathbb{B}^{b}}(x) \mid x \in M_{2}\right\}$ covers $R$, and onch $\mathbb{S}_{1 / 2}(x)$ intersects at most $n+1$ sets of $\mathfrak{U}_{2}^{\prime}$ in the same way. Hence

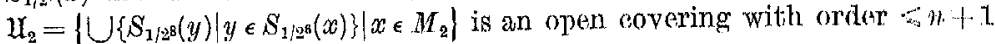
and satisfies

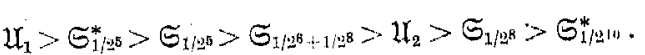

By repeating such processes we get a sequence $\mathfrak{H}_{1}>\mathfrak{U}_{2}^{*}>\mathfrak{U}_{2}>\ldots$ of open coverings of $R$ such that order $\mathfrak{U}_{m} \leqslant n+1(m=1,2, \ldots)$ and such that

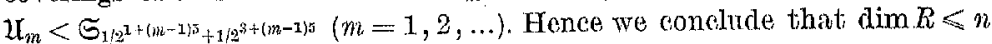
from Theorem 3 .

As is easily seen from the proof of this theorem, we can state this theorem in the following form.

Corollary 1. In order that $\operatorname{dim} R \leqslant n$ for a metrizable space $R$ it is necessary and sufficient to be able to define a metric $\varrho(x, y)$ agreeing with the topology of $R$ such that for every $\varepsilon>0$ and for some $\varphi(\varepsilon)>0$, $\varrho\left(S_{q(e)}(x), y_{i}\right)<\varepsilon(i=1, \ldots, n+2)$ imply $\varrho\left(y_{i}, y_{j}\right)<\varepsilon$ for some $i, j$ with $i \neq j$.

In the compact case we get the simpler conclusion.

COROLLARY 2 . In order that $\operatorname{dim} R \leqslant n$ for a compact metrizable space $R$ it is necessary and sufficient to be able to define a metrio $\varrho(x, y)$ agreeing with the topology of $R$ such that for every $\varepsilon>0, \varrho\left(x, y_{i}\right)<\varepsilon(i=1,2, \ldots, n+2)$ imply $e\left(y_{i}, y_{j}\right)<\varepsilon$ for some $i, j$ with $i \neq j$.

We can deduce the following theorem proved by $\mathrm{J}$. de Grooti (see [3]) from our theorem for the special case of $n=0$.

CORoI LAI.Y 3. A metrizable space $R$ is 0 -dimensional if and mly if one can define a metric which satisfies

$$
\varrho(x, z) \leqslant \max [\varrho(x, y), \varrho(y, z)] .
$$

Proof. Let $\operatorname{dim} R=0$; then by our theorem we can define a metric $\varrho(x, y)$ such that $\varrho\left(S_{e / 2}(x), y_{i}\right)<\varepsilon \quad(i=1,2)$ imply $\varrho\left(y_{1}, y_{2}\right)<\varepsilon$. Hence if we assume $\varrho(x, z)=\varepsilon>\max [\varrho(x, y), \varrho(y, z)]$ for some $x, y, z \in R$, then $\varrho\left(S_{\varepsilon / 2}(y), x\right)<\varepsilon, \varrho\left(S_{\varepsilon / 2}(y), z\right)<\varepsilon$ and $\varrho(x, z)=\varepsilon$, which contradicts the character of $\varrho(x, y)$. Therefore we must have $\varrho(x, z) \leqslant \max [\varrho(x, y)$, $\varrho(y, z)]$.
Conversely, let $\varrho(x, y)$ be a metric satisfying $\varrho(x, z) \leqslant \max [\varrho(x, y)$, $\varrho(y, z)]$, and let us assume that $\varrho\left(S_{s / 2}(x), y_{i}\right)<\varepsilon(i=1,2)$. Then there exist $x_{1}, x_{2} \in S_{\varepsilon / 2}(x)$ such that $\varrho\left(x_{i}, y_{i}\right)<\varepsilon(i=1,2)$. Since $\varrho\left(x_{1}, x_{2}\right)<\varepsilon$, we get $\varrho\left(y_{1}, y_{2}\right) \leqslant \max \left[\varrho\left(y_{1}, x_{1}\right), \varrho\left(x_{1}, y_{2}\right)\right] \leqslant \max \left[\varrho\left(y_{1}, x_{1}\right), \varrho\left(x_{1}, x_{2}\right)\right.$, $\left.\varrho\left(x_{2}, y_{2}\right)\right]<\varepsilon$.

Defristron. A real-valued function $\varrho$ of two points of a topological space $R$ is a non-Archimedean parametrio if

i) $\varrho(x, y) \geqslant 0$

ii) $\varrho(x, y)=\varrho(y, x)$,

iii) $\{y \mid \varrho(x, y)<\varepsilon\}$ is open for every $\varepsilon<0$,

iv) $\varrho(x, y) \leqslant \max [\varrho(x, z), \varrho(y, z)]$.

Now let us prove the following decomposition theorem for the metric function.

THEOREM 6. In order that $\operatorname{dim} R \leqslant n$ for a metrizable space $R$ it is necessary and sufficient to be able to define a metric $\varrho(x, y)$ agreeing with the topology of $R$ such that

$$
\begin{gathered}
\varrho(x, y)=\inf \left\{\varrho_{0}\left(x, z_{1}\right)+\varrho_{0}\left(z_{1}, z_{2}\right)+\ldots+\varrho_{0}\left(z_{p}, y\right) \mid z_{i} \in R\right\}, \\
\varrho_{0}(x, y)=\min \left\{\varrho_{i}(x, y) \mid i=1, \ldots, n+1\right\}
\end{gathered}
$$

for some $n+1$ non-Archimedean paranetrics $\varrho_{i}(x, y)(i=1, \ldots, n+1)\left({ }^{1 *}\right)$.

Proof. Necessity. Let $\operatorname{dim} R \leqslant n$; then there exist $n+10$-dimensional subspaces $R_{i}$ such that $R=\bigcup_{i=1}^{n+1} R_{i}$ by the generalized decomposition theorem. We assign a metric $\varrho^{\prime}(x, y)$ of $R$ such that $\varrho^{\prime}(x, y) \leqslant 1$. Since $R_{i}(i=1, \ldots, n+1)$ are 0 -dimensional, we get disjointed coverings $\mathfrak{U}_{m}^{i}(i=1, \ldots, n+1, m=1,2, \ldots)$ of $R_{i}$ satisfying

$$
\mathfrak{U}_{m+1}^{i}<\mathfrak{U}_{m}^{i}, \quad \mathfrak{U}_{m}^{i}<\mathfrak{S}_{m}=\left\{S_{1 / 2} m(x) \mid x \in R\right\} \quad \text { in } \quad R_{i} .
$$

Tuet $\mathfrak{L}_{m}^{i}=\left\{U_{a} \mid \alpha \in A\right\}$; then for every point $x \in R_{i}$ we can find $\alpha \epsilon A$ such that $x \in U_{z}$ and $\varepsilon(x)>0$ such that

$$
S_{\varepsilon(x)}(x) \cap R_{i} \subseteq U_{u}, \quad S_{\alpha(x)}(x) \subseteq S_{\alpha},
$$

where wo denote by $S_{\alpha}$ a definite set $\mathcal{S}_{\alpha} \in \mathbb{S}_{m+1}$ for $U_{o}$ such that $\mathcal{S}_{\alpha} \supseteq U_{\alpha}$. Put $W_{a}=\bigcup\left\{S_{s(x) / 2}(x) \mid x \in U_{\alpha} \cap R_{i}\right\}$; then we have a disjointed collection $\mathfrak{W}_{m}^{i}=\left\{W_{\alpha} \mid \alpha \in A\right\}$ satisfying $\mathfrak{W}_{m}^{i}<\widetilde{S}_{m}$. By applying $\mathfrak{B}_{m}^{i}$ we define open disjointed collections $\mathfrak{B}_{m}^{i}(i=1, \ldots, n+1, m=0,1, \ldots)$ as follows:

$$
\mathfrak{Q}_{0}^{i}=\{R\}
$$

(14) This theorem is also a generalization of the above theorem of J. 'de Groot to the ro.dimensional case. 
if $\mathfrak{B}_{m}^{i}$ is defined, then we define $\mathfrak{B}_{m+1}^{i}$ by

$$
\mathfrak{P}_{m+1}^{i}=\mathfrak{B}_{m}^{i} \wedge \mathfrak{M}_{m+1}^{i} \quad(i=1, \ldots, n+1, m=0,1, \ldots) .
$$

Then it follows that $\mathfrak{B}_{m+1}^{i}<\mathfrak{S}_{m}^{i}<\mathfrak{S}_{m}$. Now we define a real-valued function $\varrho_{i}$ of twro points by

$$
\varrho_{i}(x, y)=\inf \left\{1 / 2^{m-1} \mid y \in S\left(x, \mathfrak{B}_{m}^{i}\right)\right\} .
$$

Then it is easy to see that $\varrho_{i}$ is a non-Archimedean parametric. For $\varrho_{i}(x, y) \leqslant \max \left[\varrho_{i}(x, z), \varrho_{i}(z, y)\right]$ is evident from the disjointedness of $\mathfrak{B}_{m}^{i}$. Since

$$
S_{s}^{i}(x)=\left\{y \mid \varrho_{i}(x, y)<\varepsilon\right\}=\bigcup\left\{S\left(x, \mathfrak{B}_{i}^{i}\right) \mid \varepsilon>1 / 2^{l-1}\right\},
$$

$S_{\varepsilon}^{i}(x)$ is open for every $\varepsilon>0$. Moreover, (i) and (ii) are clearly satisfied, and hence $\varrho_{i}$ is a non-Archimedean parametric of $R$.

Since $y \in S\left(x, \mathfrak{S}_{m}^{i}\right)$ implies $\varrho^{\prime}(x, y)<1 / 2^{m-1}$ by $\mathfrak{P}_{m}^{i}<\mathbb{S}_{m}$, we have

(A) $\varrho_{i}(x, y) \geqslant \varrho^{\prime}(x, y)$ for every $x, y \in R$.

Now we can easily see that

(B) $\varrho(x, y)=\inf \left\{\varrho_{0}\left(x, z_{1}\right)+\ldots+\varrho_{0}\left(z_{p}, y\right) \mid z_{i} \in R\right\}$ $\left(\varrho_{0}(x, y)=\min \left\{\varrho_{i}(x, y) \mid i=1, \ldots, n+1\right\}\right)$

is a metric of $R$. It is enough to show only the agreement of $\varrho$ with the topology of $R$. For a given $\varepsilon>0$ and $x \in R$ we take $m$ such that $\varepsilon>1 / 2^{m-1}$ and $R_{i}$ with $x \in R_{i}, y \in S\left(x, \mathfrak{S}_{m}^{i}\right)(\neq \emptyset)$ generally implies $\varrho_{i}(x, y)<\varepsilon$ and consequently $\varrho(x, y)<\varepsilon$ by $(\mathrm{B})$. On the other hand $\varrho(x, y) \geqslant \varrho^{\prime}(x, y)$ is obvious from (A) and (B), and hence $\varrho(x, y)<\varepsilon$ implies $\varrho^{\prime}(x, y)<\varepsilon$, which proves that $\{\{y \mid \varrho(x, y)<\varepsilon\} \mid \varepsilon>0\}$ is a nbd basis of $x, i$. e., $\varrho$ agrees with the topology of $R$. Thus we deduce the necessity of the condition.

Sufficiency. Let $\varrho(x, y)$ be a metric of $R$ satisfying the condition; then we easily see that $R=\bigcup_{i=1}^{n+1} R_{i}$ if we put $R_{i}=\left\{\left.x\right|_{\varrho_{i}}(x, x)=0\right\}$. To see this we assume the existence of $x \in R$ such that $x \notin \bigcup_{i=1}^{n+1} R_{i}$. Then we must have $\varrho_{i}(x, x)=\varepsilon_{i}>0(i=1, \ldots, n+1)$, and hence by the property of $\varrho_{i}$

$$
\varrho_{i}(x, y)=\max \left[\varrho_{i}(x, y), \varrho_{i}(x, y)\right] \geqslant \varrho_{i}(x, x)=\varepsilon_{i}
$$

for every $y \in R$. Therefore $\varrho_{0}(x, y) \geqslant \min \varepsilon_{i}>0$, and hence $\varrho(x, z)$ $\geqslant \min \varepsilon_{i}>0$ for every $z \in R$, which is a contradiction.

Putting

$$
S_{1 / m}^{i}(x)=\left\{y \mid \varrho_{i}(x, y)<1 / m\right\},
$$

we see. that $S_{1 / m}^{i}(x) \cap S_{1 / m}^{i}(y) \neq \varnothing$ implies $S_{1 / m}^{i}(x)=S_{1 / m}^{i}(y)$. For, if we choose $z \in S_{1 / m}^{i}(x) \cap S_{1 / m}^{i}(y)$, then $\varrho_{i}(x, z)<1 / m, \varrho_{i}(y, z)<1 / m$, and hence $\varrho_{i}(x, y)<1 / m$ by (iv). Therefore $\varrho_{i}(y, u)<1 / m$ implies $\varrho_{i}(x, u)<1 / m$, which proves $S_{1 / n}^{i}(y) \subseteq S_{1 / m}^{i}(x)$. In the same way we get $S_{1 / m}^{i}(y) \supseteq S_{1 / m}^{i}(x)$ and consequently $S_{1 / m}^{i}(x)=S_{1 / m}^{i}(y)$. Thus

$$
\mathfrak{U}_{m}^{i}=\left\{S_{1 / m}^{i}(x) \cap R_{i} \mid x \in R_{i}\right\} \quad(n=1,2, \ldots)
$$

are open disjointed covering of $R_{i}$. Horeover, since $y \in S\left(x, \mathfrak{u}_{m}^{i}\right)$ implies $\varrho(x, y) \leqslant \varrho_{i}(x, y)<1 j m,\left\{\mathfrak{U}_{m}^{i} \mid m=1,2, \ldots\right\}$ is an open basis of $R_{i}$. Thus we conclude that $\operatorname{dim} R_{i}=0$ by Theorem 1. This combined with $R=\bigcup_{i=1}^{n+1} R_{i}$ implies $\operatorname{dim} R \leqslant n$ by the generalized decomposition theorem.

8. Imbedding of a metric space in a product of 1-dimensional spaces. We shall start with the following theorem, which is a generalization of the sufficiency part of Theorem 3.

THEOREM 7. Let $n=n_{1}+n_{2}+\ldots+n_{k}$ for non-negative integers $(i=1, \ldots, k)$. If there exist sequences

$$
\mathfrak{B}_{1, i}>\mathfrak{B}_{2, i}^{*}>\mathfrak{B}_{2, i}>\mathfrak{P}_{3, i}^{*}>\ldots \quad(i=1, \ldots, k)
$$

of open coverings of a $T_{1}$-space $R$ such that order $\mathfrak{B}_{m, i} \leqslant n_{i}+1(m=1,2, \ldots)$ and such that $\left\{S\left(p, \mathfrak{V}_{m}\right) \mid m=1,2, \ldots\right\}$ for $\mathfrak{2}_{m}=\bigwedge_{i=1}^{k} \mathfrak{B}_{m, i}$ is a nbd basis of $p$, then $R$ is a metrizable space with $\operatorname{dim} R \leqslant n$ and can be imbedded in a product of $k$ metrizable spaces $R_{i}(i=1, \ldots, k)$ with $\operatorname{dim} R_{i} \leqslant n_{i}$.

Proof. As is easily seen from the proof of Theorem 3, we can select sequences

$$
\mathfrak{U}_{1, i}>\mathfrak{U}_{2, i}^{* *}>\mathfrak{U}_{2, i}>\mathfrak{U}_{3, i}^{* *}>\ldots \quad(i=1, \ldots, k)
$$

of open coverings such that $S\left(p, \mathfrak{I}_{m+1, i}\right)$ intersects at most $n_{i}+1$ sets of $\mathfrak{U}_{m, i}$ and such that $\left\{S\left(p, \mathfrak{U}_{m}\right) ! m=1,2, \ldots\right\}$ for $\mathfrak{U}_{m}=\bigwedge_{i=1}^{k} \mathfrak{U}_{m, i}$ is a nbd basis of $p$. Luet $\mathfrak{I}_{m, i}=\left\{U_{a} \mid \alpha \in A\right\}$ for fixed $m$, $i$; then we put

(A) $V_{a}=S\left(U_{a}, \mathfrak{U}_{n+1, i}\right)$;

$$
\begin{aligned}
& W_{1,2^{m-1}}=R, \\
& W_{1,2, i}^{m}=S\left(V_{a}^{c}, \mathfrak{U}_{m+2, i}\right), \\
& W_{1 / 2^{m}+1 / 2^{m+1}}=S\left(W_{1 / 2^{m}}, \mathfrak{X}_{m+3,3, i}\right) \\
& W_{1 / i^{m+1}}=S\left(\dot{V}_{u}^{c}, \mathfrak{U}_{m+3, i}\right),
\end{aligned}
$$

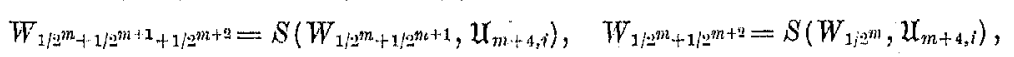

$$
\begin{aligned}
& W_{1,2^{m+1}+13^{m+8}}=S\left(W_{1,2^{m+1}}, \mathfrak{u}_{m+4, i}\right), \\
& W_{1 z^{m+3}}=S\left(V_{u}^{c}, \mathfrak{U}_{m+4, i}\right), \ldots
\end{aligned}
$$

Defining $f_{a, n, i}(x)=\inf \left\{r \mid x \in W_{r,}\right\}$, we get contimuous functions $f_{a}(a \in A)$ satisfying 
(B) $f_{a, m, i}\left(V_{a}^{c}\right)=0, \quad f_{a, m, i}\left(U_{a}\right)=1 / 2^{m-1}\left({ }^{15}\right)$.

Clearly, for every $\varepsilon>0$ there exists $l_{i}=l_{i}(\varepsilon)$ such that $y \in \mathbb{S}\left(x, \mathfrak{H}_{l_{i}, i}\right)$ implies

$$
\left|f_{a, m, i}(x)-f_{a, m, i}(y)\right|<\varepsilon \quad\left(\alpha \in A_{m, i}, m=1,2, \ldots\right) .
$$

We consider a topological product

of

$$
P_{i}=\prod\left\{I_{\alpha} \mid \alpha \in A_{m, i}, m=1,2, \ldots\right\}
$$

$$
I_{n}=\left\{x \mid 0 \leqslant x \leqslant 1 / 2^{m-1}\right\} \quad\left(a \in A_{m, i}\right),
$$

and consider $f_{a, m, i}$ a mapping of $R$ into $I_{a}$. Then we define a continuous mapping $F_{i}$ of $R$ into $P_{i}$ by

$$
F_{i}(x)=\left\{f_{a, m, i}(x) \mid \alpha \in A_{m, i}, m=1,2, \ldots\right\} \quad(x \in R) .
$$

Now we proceed to prove that $F_{i}(R)\left(\subseteq P_{i}\right)$ is a metrizable space with $\operatorname{dim} F_{i}(R) \leqslant n_{i}$. Since

$$
N_{\alpha}=F_{i}(R) \cap\left\{\left\{p_{a}\right\} \mid p_{a}>0\right\} \quad\left(\alpha \in A_{m, i}\right)
$$

are open sets, and since

$$
f_{a, m, i}\left(U_{a}\right)=1 / 2^{m}, \quad \bigcup\left\{U_{u} \mid \alpha \in A_{m, i}\right\}=R
$$

by (B), $\mathfrak{N}_{m, i}=\left\{N_{u} \mid \alpha \in A_{m, i}\right\}$ is an open covering of $F_{i}^{\prime}(R)$. First $\left\{S\left(p, \mathfrak{R}_{m, i}\right) \mid m=1,2, \ldots\right\}$ is a nbd basis of each point $p$ of $F_{i}(R)$. Let $p=\left\{p_{a} \mid a \in A_{m, i}, m=1,2, \ldots\right\} \in F_{i}(R)$; then for a given $\mathrm{nbd}$

(C) $U(p)=\left\{\left\{q_{a}\right\}|| p_{\alpha_{j}}-q_{a_{j}} \mid<\varepsilon, \alpha_{j} \in A_{m_{i}, i}(j=1, \ldots, h)\right\}$ of $p$ we choose an integer $l$ such that $y \in S\left(x, \mathfrak{U}_{l-1, i}\right)$ implies

(D) $\left|f_{a, m, i}(x)-f_{a, m, i}(y)\right|<\varepsilon\left(\alpha \in A_{m, i}, m=1,2, \ldots\right)$.

If $q \in\left\{q_{a}\right\} \in S\left(p, \mathfrak{R}_{l, i}\right)$, then $F_{i}(x)=p, F_{i}(y)=q$ and $p, q \in N_{a}$ for some $x, y \in R$ and $N_{\alpha} \in \mathfrak{R}_{l, i}$. Since

$$
f_{a, l, i}(x)=p_{a}>0, \quad f_{a, l, i}(y)=q_{u s}>0,
$$

it must be $x, y \in \nabla_{\alpha}$ for $\alpha \in A_{l, i}$. Hence it follows from (A) and $\mathfrak{U}_{l,-1, i}^{* *}<\mathfrak{U}_{l, i}$ that $y \in S\left(x, \mathfrak{U}_{l-1, i}\right)$. In consequence

$$
\left.\mid p_{a_{j}}-q_{a_{j}}\right\}=\left|f_{a_{j}, m_{j}, i}(x)-f_{u_{j}, m_{j}, i}(y)\right|<\varepsilon \quad(j=1, \ldots, h)
$$

by (D), i. e., $q \in U(p)$ follows from (C). Thus we conclude that $S\left(p, \Re_{l, i}\right)$ $\subseteq U(p)$, and hence $\left\{S\left(p, \mathfrak{N}_{m, i}\right) \mid m=1,2, \ldots\right\}$ is a nbd basis of $p$.

Text let us show that

$$
\text { order } \mathfrak{\Re}_{m, i} \leqslant n_{i}+1, \quad \mathfrak{N}_{m+1, i}^{*}<\mathfrak{R}_{m, i}
$$

(15) $f\left(T^{2}\right)=\alpha$ neans $f(x)=\alpha\left(s \in T^{5}\right)$.
If $\bigcap_{j=1}^{h} N_{\sigma_{j}} \neq \emptyset$ and $\alpha_{j} \in A_{m, i}(j=1, \ldots, h)$, then we can choose $p=\left\{p_{a,}\right\} \in F_{i}(R)$ and $x \in R$ such that

$$
\text { (E) } p \in \bigcap_{j=1}^{h} N_{a_{j}}, \quad F_{i}(x)=p .
$$

Since $f_{\alpha_{j}, n, i}(x)=p_{n_{j}}>0(j=1, \ldots, h)$ follows from $(\mathrm{E})$, we have

(F) $x \in V_{a_{j}}, \quad \alpha_{j} \in A_{m, i}(j=1, \ldots, h)$.

On the other hand, since each $S\left(p, \mathfrak{U}_{m+1, i}\right)$ intersects at most $n_{i}+1$ sets of $\mathfrak{u}_{m, i}$, we have

$$
\text { order }\left\{V_{n} \mid \alpha \in A_{m, i}\right\} \leqslant n_{i}+1
$$

from (A). Therefore we obtain $h \leqslant n_{i}+1$ from ( $\mathrm{F}$ ), which means order $\mathfrak{R}_{m, i} \leqslant n_{i}+1$.

We have learned from the above discussion that $N_{\alpha} \cap N_{a} \neq \emptyset \mathrm{im}$ plies $V_{\alpha} \cap V_{a^{\prime}} \neq \emptyset$ for fixed $m, i$ and for arbitrary $a, a^{\prime} \in A_{m+1, i}$. Hence it follows from $N_{a} \subseteq F_{i}\left(V_{a}\right)$ that

(G) $S\left(N_{n}, \mathfrak{N}_{m+1, i}\right) \subseteq F_{i}\left(S\left(V_{\alpha}, \mathfrak{V}_{m+1, i}\right)\right)$

for every $a \in A_{m+1, i}$, where we denote by $\mathfrak{B}_{m+1, i}$ the covering $\left\{V_{a} \mid a \in A_{m \mid 1, i}\right\}$ of $R$. Let $N_{a}$ be an arbitrary set of $\mathfrak{R}_{m+1, i}$, then there exists, by $\mathfrak{U}_{m+1, i}^{* *}<\mathfrak{U}_{m, i}$ $U_{\beta} \in \mathfrak{U}_{m, i}$ such that $\mathbb{S}\left(V_{a}, \mathfrak{B}_{m+1, i}\right) \subseteq U_{\beta}$. Thus we have

$$
S\left(N_{n}, \mathfrak{R}_{m+1, i}\right) \subseteq F_{i}\left(S\left(V_{n}, \mathfrak{B}_{m+1, i}\right) \subseteq \subseteq F_{i}\left(U_{\beta}\right) \subseteq N_{i} \in \mathfrak{R}_{m, i}\right.
$$

from (G) proving $\mathfrak{N}_{m+1, i}^{*}<\mathfrak{N}_{m, i}$. In consequence we can deduce from Theorem 3 the metrizability of $F_{i}(R)$ and $\operatorname{dim} F_{i}(R) \leqslant n_{i}+1$.

Now we define a mapping $F^{\prime}(x)$ of $R$ into $F_{1}(R) \times F_{2}(R) \times \ldots \times F_{k}(R)$ by

$$
F(x)=\left(F_{1}(x), \ldots, F_{k}(x)\right) \in \prod_{i=1}^{k} F_{i}(R) \quad(x \in R) .
$$

First $F^{\prime}(x)$ is one-to-one. If $x, y \in R$ and $x \neq y$, then $y \in S\left(x, \mathfrak{H}_{m, i}\right)$ for some $\mathfrak{U}_{m, i}$ because $\left\{S\left(p, \bigwedge_{i=1}^{k} \mathfrak{U}_{m, i}\right) \mid m=1,2, \ldots\right\}$ is a nbd basis of $p$. Hence $x \in U_{a} \in \mathfrak{U}_{m+1, i}, y \in V_{a}$ for some $a \in A_{m+1, i}$. This means that $f_{a, m+1, i}(x)>0$, $f_{\alpha, m+1, i}(y)=0$, i. e., $F_{i}(x) \neq F_{i}(y)$. Therefore $F^{\prime}(x)$ is one-to-one.

It remains to prove that $F^{\prime}(x)$ is homeomorphic. Since $F^{\prime}(x)$ is evidently continuous by the continuity of $E_{i}(x)$, we shall show the continuity of the inverse mapping. For a given nbd $U(x)$ of $x \in R$ we select $\mathfrak{U}_{m}=\wedge_{i=1}^{k} \mathfrak{U}_{m, i}$ satisfying $\mathbb{S}\left(x, \mathfrak{U}_{m}\right) \subseteq U(x)$. Choosing $U_{a_{i}} \in \mathfrak{U}_{m i+1, i}(i=1, \ldots, k)$ such that $x \in \bigcap_{i=1}^{k} V_{u_{i}}$, we have 
(H) $x \epsilon \bigcap_{i=1}^{k} \nabla_{a_{i}} \subseteq S\left(x, \mathfrak{U}_{m}\right) \subseteq U(x)$

from (A) and $\mathfrak{U}_{m+1, i}^{* *}<\mathfrak{U}_{m+1}$, and hence $f_{a_{i}, m+1, i}(x)=1 / 2^{m}>0$. Hence if

$$
\left|f_{a_{i}, m+1, i}(x)-f_{a_{i}, m+1, i}(y)\right|<1 / 2^{m+1} \quad(i=1, \ldots, k),
$$

then $f_{\alpha_{i}, m+1, i}(y)>0 \quad(i=1, \ldots, k)$. Therefore $y \in V_{a_{i}}(i=1, \ldots, k), i, e$. $y \in U(x)$ by $(\mathrm{H})$. This proves the continuity of $F(x)$, and consequently $R$ is homeomorphic with the subspace $F(R)$ of the product space $\prod_{i=1}^{k} F_{i}(R)$ with $\operatorname{dim} F_{i}(R) \leqslant n_{i}(i=1, \ldots, k)$. From the generalized product theorem due to Katětor and to Morita (see [4] and [5]) we have $\operatorname{dim} R \leqslant n_{1}+$ $+\ldots+n_{k}=n$.

THborem 8. Every metric space $R$ with $\operatorname{dim} R<n$ can be topologically imbedded in a topological product of $n+1$ at most 1 -dimensional metric spaces.

Proof. If $\operatorname{dim} R \leqslant n$, then it is easily shown that

(A) we can define a covering $\mathfrak{B}$ and open collections $\mathfrak{H}_{i}(i=1, \ldots, n+1)$ to every covering $\mathfrak{U}$ of $R$ such that $\mathfrak{B}<\bigcup_{i=1}^{n+1} \mathfrak{U}_{i}<\mathfrak{U}$ and such that each $S^{2}(p, \mathfrak{B})$ intersects at most one of sets belonging to $\mathfrak{U}_{i}$ for a fixed $i$. For $R=\bigcup_{i=1}^{n+1} R_{i}$ for some $R_{i}$ with $\operatorname{dim} R_{i}=0$, and hence there exists a disjointed collection $\mathfrak{B}_{i}$ of $R_{i}$ satisfying $\mathfrak{B}_{i}<\mathfrak{U}$. For every point $x$ of $R_{i}$ wede note by $\varepsilon(x)$ a positive number such that

$$
S_{\varepsilon(x)}(x) \cap R_{i} \subseteq V_{\alpha} \in \mathfrak{B}_{i}, \quad S_{e(x)}(x) \subseteq U_{a} \in \mathfrak{U}
$$

for $U_{\alpha}$ defined by $V_{a}$ so that $V_{a} \subseteq U_{\alpha}$. Then $\mathfrak{B}_{i}^{\prime}=\left\{\bigcup\left\{S_{c(x) / 2}(x) \mid x \in V_{a}\right\} \mid V_{a} \in \mathfrak{B}_{i}\right\}$ is an open collection of $R$ with $\bigcup_{i=1}^{n+1} \mathfrak{B}_{i}^{\prime}<\mathfrak{H}$. Selecting a covering $\mathfrak{B}$ with $\mathfrak{B}^{*}<\bigcup_{i=1}^{n+1} \mathfrak{B}_{i}^{i}$, we can define an open collection $\mathfrak{U}_{i}$ by

$$
\mathfrak{U}_{i}=\left\{\cup\left\{W \mid S(W, \mathfrak{D}) \subseteq V_{a}^{\prime}\right\} \mid V_{a}^{\prime} \in \mathfrak{B}_{i}^{\prime}\right\} .
$$

It is easy to see from the disjointedness of $\mathfrak{W}_{i}^{\prime}$ that $\bigcup_{i=1}^{n+1} \mathfrak{H}_{i}$ covers $R$ and that each set of $\mathfrak{W}$ intersects at most one of sets of $\mathfrak{U}_{i}$. Choosing a covexing $\mathfrak{V}$ with $\mathfrak{B}^{* *}<\mathfrak{W}$, we have open collections and a covering satisfying the required condition (A).

We denote by $\mathbb{S}_{1}>\mathfrak{S}_{2}^{*}>\mathbb{S}_{2}>\mathbb{S}_{3}^{*}>\ldots$ a sequence of coverings such that $\left\{S\left(p, \Xi_{m}\right) \mid n=1,2, \ldots\right\}$ is a nbd basis for each point of $R$, and take a corering $\mathfrak{P}$ and collections $\mathfrak{U}_{1, i}(i=1, \ldots, n+1)$ satisfying $(\mathrm{A})$ for $\mathfrak{S}_{2}, i . e$,

$$
\mathfrak{B}<\bigcup_{i=1}^{n+1} \dot{\mathfrak{U}}_{1, i}<\mathfrak{S}_{2}
$$

and $\mathfrak{S}^{2}(p, \mathfrak{B})$ intersects at most one set of $\mathfrak{U}_{1, i}$. Let $\mathfrak{H}_{1, i}=\left\{U_{\alpha} \mid \alpha \in A\right\}$ and define $\mathfrak{N}_{1, i}$ by

$$
\mathfrak{N}_{1, i}=\left\{S\left(U_{a}, \mathfrak{B}\right), R-\bigcup_{a \in, A} \vec{U}_{\alpha} \mid \alpha \in A\right\} \quad \text { for a fixed } i
$$

then $\mathfrak{N}_{1, i}$ is a covering of order $\leqslant 2$. Moreover, it follows from $\mathcal{S}_{2}^{*}<\subseteq_{1}$ and $\bigcup_{i=1}^{n+1} \mathfrak{U}_{1, i} \supseteq R$ that $\bigwedge_{i=1}^{n+1} \mathfrak{N}_{1, i}<\Im_{1}$.

Now we notice that

(B) every covering $\mathfrak{P}$ of order $\leqslant 2$ has a locally finite star-refinement $Q^{\prime}$ with order $\leqslant 2$.

To show this we put $\mathfrak{P}=\left\{P_{\delta} \mid \delta \in D\right\}$ and denote by $\mathfrak{P}^{\prime}$ a star-refinement of $\mathfrak{B}$. Then

(C) $\mathfrak{M}=\left\{M_{\delta}=\bigcup\left\{P^{\prime} \mid \mathbb{S}\left(P^{\prime}, \mathfrak{P}^{\prime}\right) \subseteq P_{\delta}, P^{\prime} \in \mathfrak{B}^{\prime}\right\} \mid \delta \in D\right\}$

is a locally finite refinement of $\$$ of order $\leqslant 2$. We define an open set $t_{\delta}$ for every $\delta \in D$ such that

(D) $M_{\delta}-\bigcup_{\delta \neq \delta^{\prime} \in D} M_{\delta^{\prime}} \subseteq L_{\delta} \subset \vec{L}_{\delta} \subseteq M_{\delta}$ and put

(E) $Q_{\delta}=L_{\delta}-\bigcup_{\delta^{\prime} \neq \delta} \bar{L}_{\delta^{\prime}}, \mathbb{Q}=\left\{Q_{\delta}, M_{\alpha} \cap X_{\beta} \mid \delta, \alpha, \beta \in D, \alpha \neq \beta\right\}$.

It is easy to see that $Q$ is an open covering satisfying $Q^{1}<\$\left({ }^{16}\right)$ order $Q<2$. To show that $Q$ covers $R$ we take an arbitrary point $p$ of $R$. If $p \in M_{s}$ and $p \notin M_{\delta^{\prime}}\left(\delta^{\prime} \neq \delta\right)$, then $p \in L_{\delta}$ by (D). Since $\left\{\bar{L}_{\delta} \mid \delta \in D\right\}$ is locally finite, it follows from (D) that there exists a $\operatorname{nbd} U(p)$ of $p$ such that $U(p) \cap L_{\delta^{\prime}}=\varnothing$ for every $\delta^{\prime}: \delta^{\prime} \neq \delta$. Hence $p \notin \underset{\delta^{\prime} \neq \delta}{\bigcup} \overline{L_{\delta^{\prime}}}$ and consequently $p \in Q_{\delta}$ by (E). Therefore from (C), (D), (E) we get that $\mathbb{Q}$ covers $R$ and that $S(p, \mathbb{Q})$ $=Q_{\delta} \subseteq M_{\delta} \subseteq P_{\delta}$. If $p \in M_{a} \cap M_{\beta}$, then $p \notin M_{\gamma}$ for every $\gamma$ with $\gamma \neq \alpha, \beta$. It follows from (E) that either $p \notin Q_{a}$ or $p \dot{\xi} Q_{\beta}$. Therefore either

$01^{*}$

$$
\begin{aligned}
& S(p, \mathfrak{Q})=Q_{a} \cup\left(M_{a} \cap M_{\beta}\right) \subseteq M_{a} \subseteq P_{\alpha} \\
& S(p, \mathbb{Q})=Q_{\beta} \cup\left(M_{u} \cap M_{\beta}\right) \subseteq M_{\beta} \subseteq P_{a}
\end{aligned}
$$

which shows that $\mathfrak{Q}^{d}<\mathfrak{F}$ and order $\mathbb{Q} \leqslant 2$. Repeating such a process we have a locally finite $\triangle$-refinement $Q^{\prime}$ of $\mathbb{Q}$ with order $\leqslant 2$. $\mathbb{Q}^{\prime}$ satisfies the required condition of (B).

To show the existence of sequences $\mathfrak{N}_{1, i}>\mathfrak{N}_{2, i}^{*}>\mathfrak{N}_{2, i}>\mathfrak{N}_{3, i}^{*}>\ldots$ $(i=1, \ldots, n+1)$ of coverings of order $\leqslant 2$ such that $\bigwedge_{i=1}^{n+1} \mathfrak{N}_{m, i}<\mathbb{S}_{m}$,

('ib) $Q^{4}=\{S(p, Q) \mid p \in R\}$.

Fundamenta Mathematicae, T. XLV. 
we assume the existence of such $\mathfrak{N}_{l, i}$ for $l \leqslant m$. Then there exists by (B) a locally finite covering $\mathfrak{N}_{i}$ of order $\leqslant 2$ such that $\mathfrak{N}_{i}^{*}<\mathfrak{N}_{m, i}$. Let us select a covering $\mathfrak{M}$ with $\mathfrak{M}^{* *}<\wedge_{i=1}^{n+1} \mathfrak{N}_{i}$. Next we select by (A) a covering $Q$ and open collections $\mathfrak{P}_{i}(i=\stackrel{i=1}{1}, \ldots, n+1)$ such that

(F) $Q<<\bigcup_{i=1}^{n+1} \mathfrak{P}_{i}<\mathfrak{M} \wedge \mathfrak{S}_{m+2}$

"and such that each $S^{2}(p, \mathbb{Q})$ intersects at most one of sets belonging to $\mathfrak{P}_{i}$ for a fixed $i$. We put $\mathfrak{P}_{i}=\left\{P_{\beta} \mid \beta \in B\right\}, \mathfrak{R}_{i}=\left\{N_{\gamma} \mid \gamma<\tau\right\}$ and denote by $\gamma(\beta)$ the first ordinal $\gamma$ satisfying

(G) $\overline{S\left(P_{\beta}, \mathfrak{Q}\right)} \subseteq N_{\gamma} \in \mathfrak{N}_{i}$

for $\beta \in \mathcal{B}$. Then we define a covering $\mathfrak{R}_{m+1, i}$ by

(H) $\mathfrak{N}_{m+1, i}=\left\{K_{\gamma}, S\left(P_{\beta}, \mathbb{Q}\right) \mid \gamma<\tau, \beta \in B\right\}$,

where we put

(I) $\bar{K}_{\gamma}=N_{\gamma}-\bigcup\left\{\bar{P}_{\beta} \mid \gamma=\gamma(\beta)\right\} \cup\left\{\overline{S\left(\bar{P}_{\beta}, \mathfrak{Q}\right)} \mid \gamma \neq \gamma(\beta)\right\}$.

It easily follows that

(J) $\mathfrak{N}_{m+1, i}<\mathfrak{N}_{i}$ and order $\mathfrak{N}_{m+1, i} \leqslant 2$.

Since $\mathfrak{R}_{m+1, i}<\mathfrak{N}_{i}$ is obvious, let us prove the latter assertion. We denote by $x$ an arbitrary point of $R$. First we consider the case of $x \notin S\left(P_{\beta}, \mathbb{Q}\right)(\beta \in B)$. Then either $x \in \overline{S\left(P_{\beta}, \mathcal{Q}\right)}$ for some $\beta \in B$ or $x \notin \overline{S\left(P_{\beta}, Q\right)}$ for every $\beta \in B$. If the former is the case, then $x \notin \overline{S\left(P_{\beta^{\prime}}, \mathbb{Q}\right)}$ for every $\beta^{\prime}$ with $\beta^{\prime} \neq \beta$ because $S^{2}(x, \mathfrak{Q})$ intersects at most one set of $\mathfrak{P}_{i}$. Hence it follows from (G), (I) that $x \in K_{\gamma(\beta)}$ and $x \notin K_{\gamma}(\gamma \neq \gamma(\beta))$. If the latter is the case, then it follows from (I) and order $\mathfrak{R}_{i} \leqslant 2$ that $x \in K_{\gamma}$ for some (at most two) $\gamma$ such that $x \in N_{\gamma}$. Next we consider the case of $x \in S\left(P_{\beta}, \mathbb{Q}\right)$. Then $x \notin S\left(P_{\beta^{\prime}}, Q\right)$ for every $\beta^{\prime}$ with $\beta \neq \beta^{\prime}$ as in the above discussion. Since $x \notin K_{\gamma}$ for $\gamma \neq \gamma(\beta)$ is obvious from (G), by (H) $x$ is contained in at most two sets of $\mathfrak{N}_{m+1, i}$. Thus in every case $x$ is contained in at most two sets of $\mathfrak{N}_{m+1, i}, i . e ., \mathfrak{N}_{m+1, i}$ is a covering of order $\leqslant 2$.

Let $K_{\gamma_{i}} \in \mathfrak{N}_{m+1, i}(i=1, \ldots, n+1)$ and let $x$ be an arbitrary point of $R$; then $x \in P_{\beta} \in \mathfrak{B}_{i}$ for some $i$ because $\bigcup_{i=1}^{n+1} \mathfrak{P}_{i}$ covers $R$. Hence $x \notin K_{\gamma_{i}}$ by (I), which shows $\bigcap_{i=1}^{n+1} K_{\gamma_{i}}=\emptyset$. Therefore every set of $\bigwedge_{i=1}^{n+1} \mathfrak{N}_{m+1, i}$ is contained in $S\left(P_{\beta}, \mathfrak{Q}\right)$ with $P_{\beta} \in \mathfrak{P}_{i}$ for some $i$, which implies

(K) $\bigwedge_{i=1}^{n+1} \mathfrak{N}_{m+1, i}<\left(\bigvee_{i=1}^{n+1} \mathfrak{B}_{i}\right)^{*}<\Im_{m+2}^{*}<\varsigma_{m+1}$

by (F). It follows from (J) and $\mathfrak{N}_{i}^{*}<\mathfrak{N}_{m, i}$ that $\mathfrak{N}_{m+1, i}^{*}<\mathfrak{N}_{m, i}$. This combined with $(J),(K)$ completes the induction, and hence we get sequences

$$
\mathfrak{N}_{1, i}>\mathfrak{N}_{2, i}^{*}>\mathfrak{N}_{2, i}>\mathfrak{N}_{3, i}^{*}>\ldots . \quad(i=1, \ldots, n+1)
$$

such that $\bigwedge_{i=1}^{n+1} \mathfrak{N}_{m, i}<\mathfrak{S}_{m}$, order $\mathfrak{N}_{m, i} \leqslant 2$. Hence by Theorem 7 we can imbed $R$ into a topological product of $n+1$ metrizable spaces $R_{i}$ with $\operatorname{dim} R_{i} \leqslant 1$.

\$ 4. Imbedding $n$-dimensional spaces in $E_{2 n+1} \times N(\Omega)$.

DefiNition. We call a covering $\mathfrak{U}$ star-finite (star-countable) if every set of $\mathfrak{U}$ intersects finitely (countably) many sets of $\mathfrak{H}$.

An open basis consisting of an enumerable number of star-finite (star-countable) open coverings is called a $\sigma$-star-finite ( $\sigma$-star-countable) open basis.

Remark. A regular space $R$ has a $\sigma$-star-finite basis if and only if $R$ has a $\sigma$-star-countable basis. Moreover $\mathrm{K}$. Morita has proved the following theorem: A regular space having a $\sigma$-star-finite ( $\sigma$-star-countable) basis can be imbedded in the topological product $N(\Omega) \times I^{\omega}$ of a generalized Baire 0 -dimensional space $N(\Omega)\left({ }^{17}\right)$ and Hilbert cube $I^{\omega}$, and the converse is also true.

Remark. A metric space having a $\sigma$-star-finite basis need not have the star-finite property or the star-countable property (18). For example, $N(\Omega) \times\{x \mid 0<x<1\}$ has obviously a $\sigma$-star-finite basis, but it has not the star-countable propetry if the cardinal number of $\Omega$ is greater than $s_{0}$. For if we put

$$
S\left(a_{1}, \alpha_{2}, \ldots, \alpha_{k}\right)=\left\{p \mid p=\left(\alpha_{1}, \alpha_{2}, \ldots, \alpha_{k}, \ldots\right) \in N(\Omega)\right\},
$$

then it is easily seen that the open covering

$$
\begin{gathered}
\left\{N(\Omega) \times\{x \mid 1 / 2<x<1\}, S\left(\alpha_{1}\right) \times\left\{x \mid 1 / 2^{2}<x<1 / 2+1 / 2^{2}\right\}, \ldots, S\left(\alpha_{1}, \ldots, \alpha_{k}\right) \times\right. \\
\left.\times\left\{x \mid 1 / 2^{k+1}<x<1 / 2^{k}+1 / 2^{l k+1}\right\}, \ldots \mid \alpha_{i} \in \Omega(i=1,2, \ldots)\right\}
\end{gathered}
$$

of this space has no star-countable refinement and accordingly no starfinite refinement. To see this we assume that $\mathfrak{U}$ is a star-countable refinement of this covering. Then $\bigcup_{n=1}^{\infty} S^{n}(U, \mathfrak{U})$ for an arbitrary $U \in \mathfrak{X}$ consisting of countably many sets of $\mathfrak{U}$. We can select $S\left(\alpha_{1}, \ldots, \alpha_{k}\right) \times I \subseteq U$. It follows from the connectedness of $\{x \mid 0<x<1\}$ that

$$
\bigcup_{n=1}^{\infty} S^{n}(U, \mathfrak{U}) \supseteq S^{n}\left(\alpha_{1}, \ldots, \alpha_{k}\right) \times\{x \mid 0<x<1\} .
$$

${ }^{\left({ }^{12}\right)}$ This notion is due to [5]. For any two sequences of elements from an abstract set $\Omega \alpha=\left(\alpha_{1}, \alpha_{2}, \ldots\right), \beta=\left(\beta_{1}, \beta_{2}, \ldots\right)$, we define the metric $d^{\prime}(\alpha, \beta)$ by

$$
d^{\prime}(\alpha, \beta)=1 / \min \left\{k \mid \alpha_{k} \neq \beta_{k}\right\}, \quad d^{\prime}(\alpha, \alpha)=0 .
$$

Then the set $N(\Omega)$ of all such sequences turns out to be a zero-dimensional space.

(18) We say that $R$ has the star-finite (star-countable) property if only if every open covering of $R$ has a star-finite (star-countable) open refinement. 
Hence $\bigcup_{n=1}^{\infty} S^{n}(U, \mathfrak{X})$ contains every set of $\mathfrak{U}$ contained in $S\left(\alpha_{1}, \ldots, a_{k}, a_{k+1}\right) \times$ $\times\left\{x \mid 1 / 2^{k+2}<x<1 / 2^{k+1}+1 / 2^{k+2}\right\}$ for some $\alpha_{k+1} \in \Omega, \quad$ i. e.., $\bigcup_{n=1}^{\infty} S^{n}(U, \mathfrak{U})$ contains non-enumerably many sets of $\mathfrak{U}$, which is a contradiction.

THEOREM 9. Suppose that $R$ is a regular space having a $\sigma$-star-finite $(\sigma-$ star-countable) basis and $\operatorname{dim} R \leqslant n$. Then $R$ is homeonorphic to a subset of $N(\Omega) \times I_{2 n+1}$, where $I_{2 n+1}$ is a $(2 n+1)$-dimensional Euclidean cube and $N(\Omega)$ is the generatized Baire 0 -dimensional space for a set $\Omega$ whose cardinal number is not less than the cardinal number of an open basis of $R$.

Proof. 1. There exists, as is seen from the above Morita's theorem, a sequence $\mathfrak{N}_{1}>\mathfrak{N}_{2}>\mathfrak{N}_{3}>\ldots$ of star-finite open coverings $\mathfrak{N}_{m}$ of $R$ such that $\left\{S\left(p, \mathfrak{N}_{m}\right) \mid m=1,2, \ldots\right\}$ is a $\mathrm{nbd}$ basis of every point $p$ of $R$. We define a disjointed covering $\Xi_{m}$ of $R$ by $\Xi_{m}=\left\{S^{\infty}\left(N, \mathfrak{N}_{m}\right) \mid N \in \mathfrak{N}_{m}\right\}$, where $S^{\infty}\left(N, \mathfrak{N}_{m}\right)=\bigcup_{n=1}^{\infty} S^{n}\left(N, \mathfrak{N}_{m}\right)$. Let $\mathfrak{S}_{m}=\left\{S_{\alpha} \mid \alpha \in A_{m}^{\prime}\right\}$ and $S_{\alpha} \cap S_{\beta}=\varnothing(\alpha \neq \beta)$; then for every $\alpha \epsilon A_{m} S_{\alpha}$ is a countable sum of sets of $\mathfrak{R}_{m}^{z}$, i. e.,

$$
S_{a}=\bigcup\left\{N_{a, i}^{(m)} \mid i=1,2, \ldots\right\}, \quad N_{a, i}^{(m)} \in \mathfrak{N}_{n} \quad(i=1,2, \ldots)^{n}
$$

Siuce $\mathfrak{N}_{m}$ is locally finite, there exists an open covering $\mathfrak{P}_{m}$ of $R$ such that

$$
\mathfrak{P}_{m}=\left\{P_{a, i}^{(m)} \mid a \in A_{m}, i=1,2, \ldots\right\}, \quad \overline{P_{a, i}^{(m)}} \subseteq N_{a, i}^{(m)} .
$$

Next, we define a sequence of open coverings by

$$
\mathfrak{U}_{i, m}=\left\{N_{a, i}^{(m)}, \$_{a}-\overline{P_{a, i}^{(m)}} \mid \alpha \in A_{m}\right\},
$$

$\mathfrak{H}_{\mathbf{I}}=\mathfrak{U}_{1,1}, \mathfrak{U}_{2}=\mathfrak{U}_{1} \wedge \mathfrak{U}_{2,1} \wedge \mathfrak{U}_{1,2}, \ldots, \mathfrak{U}_{m}=\mathfrak{U}_{m-1} \wedge \mathfrak{U}_{m, 1} \wedge \mathfrak{U}_{m-1,2} \wedge \ldots \wedge \mathfrak{U}_{1, m}, \ldots$

Then $\mathfrak{U}_{1}>\mathfrak{U}_{2}>\mathfrak{U}_{3}>\ldots$, and $\left\{S\left(p, \mathfrak{U}_{m}\right) \mid m=1,2, \ldots\right\}$ is a nbd basis of each point $p$ of $R$, and

(A) $\mathfrak{U}_{m}$ is finite in every $S_{a}\left(\alpha \in A_{k}, k \geqslant m\right)$.

Let $\Omega=\bigcup_{n=1}^{\infty} A_{m}$; then it is clear from the disjointedness of $\mathfrak{S}_{m}$ that $|Q| \leqslant$ the cardinal number of any open basis of $R$. We define a continnous mapping $o(x)$ of $R$ into $N(\Omega)$ by

$$
e(x)=\left(\alpha_{1}, \alpha_{2}, \ldots\right) \quad\left(x \in \mathbb{S}_{\alpha_{m}}, a_{m} \in A_{m} ; m=1,2, \ldots\right)
$$

and denote by $M(R)$ the totality of a continuous mapping $\varphi$ of $R$ into $N(\Omega) \times I_{2 n+1}$ such that $\varphi(x)=(c(x), \Phi(x))(x \in R)$ for a continuous mapping $\Phi(x)$ of $R$ into $I_{2 n+1}$.
Moreover we define the following notions, which will be needed later on.

$$
\begin{aligned}
& T_{a}=c\left(S_{a}\right)=\left\{\left(\alpha_{1}, a_{2}, \ldots\right) \mid a_{m}=\alpha\right\} \cap c(R) \quad \text { for } \quad a \in A_{m}, \\
& \mathfrak{I}_{a}=\left\{T_{\alpha} \times S_{1 / m}(x)\left\{x \in I_{2 n+1}\right\}\right.
\end{aligned}
$$

where we denote by $S_{1 / m}(x)$ the spherical nbd of radius $1 / m$ around $x$ in $X_{2 n+1}$. We mean by a star-decomposition a disjointed covering of $R$ consisting of open sets contained in $\bigcup_{m=1}^{\infty} \mathfrak{S}_{m}$. Let $C:\left\{S_{:,} ; \gamma \in C\left(\subseteq \bigcup_{m=1}^{\infty} A_{m}\right)\right\}$ be a star-decomposition; then for every $\gamma \in C$ we denote by $m(\gamma)$ such a number that $\gamma \in A_{m(\gamma)}$,

We denote by $M(R, m)$ the totality of mappings of $M(R)$ satisfying

$$
f^{-1}\left(\mathfrak{I}_{\gamma}\right)=\left\{f^{-1}(T) \mid T \in \mathfrak{I}_{\eta}\right\}<\mathfrak{U}_{m} \quad(\gamma \in C)
$$

for some star-decomposition $C:\left\{S_{,} ; \gamma \in G\right\}$ of $R$. Finally we define $O$-neighborhood $N_{C}(f)$ of $f \in M(R)$ by

$$
N_{C}(f)=\left\{g \mid g \in M(R), \sup \left\{d(\mid \pi f(x), \pi g(x)) \mid x \in S_{g}\right\}<1 / m(\gamma)\right\}
$$

for a star-decomposition $G:\left\{S_{\psi} \mid \gamma \in C\right\}$, where $\pi$ and $d$ denote the projection of $N(\Omega) \times I_{2 n+1}$ onto $I_{2 n+1}$ and the metric of $I_{2 n+1}$ respectively.

2. First we prove

(B) $N_{C}(f) \cap M(R, m) \neq \varnothing$ for every $f \epsilon M(R)$, every star-decomposition $C$ and every positive integer $m$.

Take $l(\gamma)=\max (6 m(\gamma), m)$ for every $\gamma \in O$ and put

$$
D_{\gamma}=\left\{\delta \mid \delta \in A_{l\left(i^{\prime}\right)}, T_{\delta} \subseteq T_{;} \text {(or } S_{\delta} \subseteq S_{,} \text {, as the same) }\right\} \text {. }
$$

Since we can cover $I_{2 n+1}$ by a finite subcovering of $\left\{S_{1 / n(\gamma)}(x) \mid x \in I_{2 n+1}\right\}$, we denote by $\left\{S_{1 / l\left(l^{\prime}\right)}\left(\infty_{i}\right) \mid i=1,2, \ldots, a(\gamma)\right\}$ such a covering; then $\mathfrak{I}_{\delta}^{\prime}=\left\{T_{\delta} \times \mathbb{S}_{1 /\left(l_{(\gamma)}\right)}\left(x_{i}\right) \mid i=1,2, \ldots, a(\gamma)\right\}$ is a finite subcovering of $\mathfrak{I}_{\delta}=\left\{T_{\delta} \times S_{1 / l(\gamma)}(x) \mid x \in I_{2 n+1}\right\}\left(\delta \in A_{l(\gamma)}\right)$. Since $f^{-1}\left(\mathfrak{I}_{\delta}^{\prime}\right)=\left\{f^{-1}\left(T^{\prime}\right) \mid T^{\prime} \in \mathfrak{I}_{\delta}^{\prime}\right\}$ and $\mathfrak{U}_{m}$ are, by $l(\gamma) \geqslant m$ and $(A)$, finite open coverings of $S_{\delta}$, we have an open finite covering $\mathfrak{B}_{\delta}$ of $\mathbb{S}_{\delta}$ satisfying order $\mathfrak{B}_{\delta} \leqslant n+1, \mathfrak{B}_{\delta}^{d}<\mathfrak{U}_{m} \wedge f^{-1}\left(\mathfrak{I}_{\delta}^{\prime}\right)$. $\mathfrak{B}=\bigcup\left\{\mathfrak{B}_{\delta} \mid \delta \in D_{\gamma}, \gamma \in C\right\}$ is an open covering of $R$ of order $\leqslant n+1$.

Let us consider fixed $\gamma \in C$ and $\delta \in D_{y}$, and assume that $V_{1}, \ldots, V_{s}$ are all the numbers of $\mathfrak{B}_{\delta}$. Then we select vertices $x\left(V_{i}\right)(i=1, \ldots, s)$ in $I_{2 n \div 1}$ for which it is true that $d\left(\pi f\left(V_{i}\right), x\left(\nabla_{i}\right)\right)<1 / 3 m(\gamma)(i=1, \ldots, s)$, the $x\left(V_{i}\right)$ are in a general position in $E_{2 n+1}$, i. e., no $m+2$ of the vertices $x\left(V_{i}\right)(m=0,1, \ldots, 2 n)$ lie in an $m$-dimensional linear subspace of $E_{2 n+1}$. We define a barycentric mapping $\Phi_{\delta}$ of $S_{\delta}$ into $I_{2 n+1}$ by 
(C) $\Phi_{\delta}(p)=\frac{\sum_{i=1}^{s} \varrho\left(p, \nabla_{i}^{c}\right) x\left(\nabla_{i}\right)}{\sum_{i=1}^{s} \varrho\left(p, \nabla_{i}^{c}\right)} \quad\left(p \in S_{\delta}\right)$,

where we consider $x\left(V_{i}\right)(i=1, \ldots, s)$ as vectors and denote by

$$
\varrho\left(x, V_{i}^{c} \inf \left\{\varrho(p, q) \mid q \in \nabla_{i}^{c}\right\}{ }^{(19)} .\right.
$$

Thus we get a continuous mapping

$$
\Phi(p)=\Phi_{\delta}(p) \quad\left(p \in S_{\delta}, \delta \in D_{\gamma}, \gamma \in C\right)
$$

of $R$ into $I_{2 n+1}$. We now prove that the mapping $\varphi(p)=(e(p), \Phi(p)) \in M(R)$ is contained in the common part of $N_{C}(f)$ and $M(R, m)$.

To prove $\varphi \in N_{C}(f)$ we take an arbitrary point $p \in S_{\gamma}$ for $\gamma \in C$. Then $p \in S_{\delta}$ for some $\delta \in D_{\gamma}$. Assume that $V_{i}$ are so numbered that $\left\{V_{1}, \ldots, V_{l}\right\}$ is the set of all the $\nabla_{i} \in \dot{\mathfrak{B}}_{\delta}$. which contain $p$. Then $\varrho\left(p, \nabla_{i}^{c}\right)=0$ for $i>t$. From we get

$$
\delta\left(\pi f\left(\nabla_{i}\right)\right) \leqslant 2, l(\gamma) \leqslant 1,3 m(\gamma) \quad \text { and } \quad d\left(\pi f\left(V_{i}\right), x\left(\nabla_{i}\right)\right)<1 / 3 m(\gamma)
$$

$$
a\left(x\left(\nabla_{i}\right), \pi f(p)\right)<2 / 3 m(\gamma) \quad(i=1,2, \ldots, t) .
$$

A fortiori, the centre of gravity $\Phi(p)$ of the $x\left(Y_{i}\right)$ satisfies

$$
d(\Phi(p), \pi f(p))<2 / 3 m(\gamma)<1 / m(\gamma) .
$$

Therefore $\varphi \in N_{C}(f)$.

Next in order to show that $\varphi \in M(R, m)$ we fix $\gamma \in O$ and $\delta \in D_{\gamma}$ and suppose that $V_{i_{1}}, \ldots, V_{i_{i}}$ are all the members of $\mathfrak{B}_{\delta}$ containing a given point $p$ of $S_{\delta}$. Consider the linear $(t-1)$-space $L_{\delta}(x)$ in $I_{2 n+1}$ spanned by the vertices $x\left(\nabla_{i_{1}}\right), \ldots, x\left(\nabla_{i_{b}}\right)$; then $t \leqslant n+1$ and $\Phi_{\delta}(p) \in L_{\delta}(p)$ are obrious from (C).

Since there are only a finite number of linear subspaces $L_{\delta}(p)$, there exists a positive number $h(\delta)>l(\delta)$ such that any two of these linear subspaces $L_{\delta}(p)$ and $L_{\delta}\left(p^{\prime}\right)$ either meet or are at a distance $\geq 2 / h(\delta)$ from each other.

Putting $\mathbb{E}_{\delta}=\left\{\varepsilon \mid \varepsilon \in A_{h(\delta)}, T_{\varepsilon} \subseteq T_{\delta}\right\}$, we consider a star-decomposition

$$
E:\left\{S_{\varepsilon} \mid \varepsilon \in E_{\delta}, \delta \in D_{\gamma}, \gamma \in O\right\} .
$$

If $\varphi(p), \varphi\left(p^{\prime}\right) \in T_{c} \times \beta_{1 / \hbar(\delta)}(x)$ for $p, p^{\prime} \in R$, then it follows that $c(p)$, $e\left(p^{\prime}\right) \in T_{\varepsilon}$ and

$$
d(\Phi(p), x)<1 / h(\delta), \quad d\left(\Phi\left(p^{\prime}\right), x\right)<1 / h(\delta) ;
$$

(iv) $\varrho(p, q)$ denotes the metric of $R$. hence $p, p^{\prime} \in S_{\varepsilon} \subseteq S_{\delta}$. Therefore we get $d\left(\Phi(p), \Phi\left(p^{\prime}\right)\right)<2 / h(\delta)$, which implies $L_{\delta}(p) \cap \bar{L}_{\delta}\left(p^{\prime}\right) \neq \emptyset$. If we suppose that $L_{\delta}\left(p^{\prime}\right)$ is spanned by $x\left(\nabla_{j_{1}}\right), \ldots, x\left(V_{j_{u}}\right), u \leqslant n+1$, then since $x\left(V_{i_{2}}\right), \ldots, x\left(V_{i_{i}}\right), x\left(\nabla_{j_{1}}\right), \ldots, x\left(\nabla_{j_{u}}\right)$ are in a general position in $E_{2 n+1}$, it follows that at least one of $x\left(\nabla_{j_{1}}\right), \ldots, x\left(\nabla_{j_{u}}\right)$ is also one of $x\left(\nabla_{i_{z}}\right), \ldots, x\left(\dot{\nabla}_{i_{v}}\right)$. Hence $p$ and $p^{\prime}$ are contained in a common member $\nabla_{i}$ of $\mathfrak{B}_{\delta}, i . e_{.}, p^{\prime} \in S\left(p, \mathfrak{B}_{\delta}\right)$. It follows from $\mathfrak{O}_{\delta}^{A}<\mathfrak{U}_{m}$ that $\varphi^{-1}\left(T_{\varepsilon} \times S_{1 / h(\delta)}(x)\right) \subseteq U$ for some $U \in \mathfrak{U}_{m}$. Thus we get $\varphi^{-1}\left(\mathfrak{I}_{\varepsilon}\right)<\mathfrak{U}_{m}$ for every $\varepsilon \in E$, proving $\varphi \in M(R, m)$. We now prove that

(D) for a given $\varphi \in N_{C}(f) \cap M(R, m)$ there exists a star-decomposition $C^{\prime}$ satisfying $N_{C^{\prime}}(\varphi) \subseteq N_{C}(f) \cap M(R, m)$.

Since $\varphi \in N_{C}(f)$ implies

$$
\sup \left\{d(\pi f(p), \pi \varphi(p)) \mid p \in S_{\gamma}\right\}=\alpha_{\gamma}<1 / m(\gamma) \quad(\gamma \in C),
$$

we take a positive integer $h(\gamma)$ for $\gamma \in C: 1 / h(\gamma)<1 / m(\gamma)-a_{\gamma}$ and define a star-decomposition $D$ by

$$
\text { - } D:\left\{S_{\delta} \mid \delta \in D_{\gamma}, \gamma \in O\right\} \quad\left(D_{\gamma}=\left\{\delta \mid \delta \in A_{h(\gamma)}, T_{\delta} \subseteq T_{\gamma}\right\}\right) .
$$

Let $\psi \in N_{D}(\varphi)$; then taking $p \in S_{\delta}, \delta \in D_{\gamma}$ for a given point $p$ of $S_{\gamma}$, we get $d(\pi \varphi(p), \pi \psi(p))<1 / h(\gamma)$. Therefore

$$
\sup \left\{d(\pi f(p), \pi \psi(p)) \mid p \in S_{\gamma}\right\} \leqslant a_{\gamma}+1 / h(\gamma)<1 / m(\gamma),
$$

proving $\psi \in N_{C}(f)$, i. e., it holds that

(E) $N_{D}(\varphi) \subseteq N_{C}(f)$.

Moreover, since $\varphi \in M(R, m)$, we have $\varphi^{-1}\left(\mathfrak{I}_{\beta}\right)<\mathfrak{U}_{m}(\beta \in B)$ for some star-decomposition $B$, where $\mathfrak{I}_{\beta}=\left\{T_{\beta} \times S_{1 / m(\beta)}(x) \mid x \in I_{2 n+1}\right\}$ as above defined. Putting $D_{\beta}=\left\{\delta \mid \delta \in A_{2 m(\beta)}, T_{\delta} \subseteq T_{\beta}\right\}$ for every $\beta \in B$, we have a stardecomposition $E:\left\{S_{\varepsilon} \mid \varepsilon \in D_{\beta}, \beta \in B\right\}$. Let $\psi \in N_{E}(\varphi)$; then we easily see that $\varepsilon \in D_{\beta}$ implies

$$
\psi^{-1}\left(T_{s} \times S_{1 / 2 m(\beta)}(x)\right) \subseteq \varphi^{-1}\left(T_{\beta} \times S_{1 / m(\beta)}(x)\right)
$$

for every $x \in I_{2 n+1}$. For if we assume the contrary, then there exists a point $p$ of $R$ such that

$$
\psi(p) \in X_{8} \times S_{1 / 2 m(\beta)}(x), \quad \varphi(p) \notin T_{\beta} \times S_{1 / m(\beta)}(x) .
$$

Hence $p \in S_{s}, \pi \psi(p) \in S_{1 / 2 m(\beta)}(x)$ and $\pi \varphi(p) \notin S_{1 / m(\beta)}(x)$, and hence $d(\pi \psi(p), \pi \varphi(p)) \geqslant 1 / 2 m(\beta)$, which contradicts $\psi \in N_{\varepsilon}(\varphi)$. Thus we must have

$$
\psi^{-1}\left(T_{s} \times S_{1 / 2 m(\beta)}(x)\right) \subseteq \varphi^{-1}\left(T_{\beta} \times S_{1 / m(\beta)}(x)\right) \subseteq U
$$

for some $U \in \mathfrak{U}_{m}$. Therefore $\psi^{-\mathrm{d}}\left(\mathfrak{T}_{e}\right)<\mathfrak{H}_{m}(\varepsilon \in E)$, proving $\psi \in M(R, m)$, i. e., $N_{E}(\varphi) \subseteq M(R, m)$. This combined with (E) shows that $U^{\prime}=D \wedge E$ 
$=\left\{S_{\delta} \cap S_{\varepsilon} \mid \delta \in D, \varepsilon \in E\right\}$ is a star-decomposition satisfying $N_{C^{\prime}}(\varphi) \subseteq N_{C}(f)$ $\cap M(R, m)$.

3. We can select by (B) and (D) two sequences $O_{1}>C_{2}>O_{3}>\ldots$, $D_{1}>D_{2}>D_{3}>\ldots$ of star-decompositions and a sequence $f_{1}, f_{2}, f_{3}, \ldots$ of elements of $M(R)$ such that

$$
\begin{gathered}
\gamma \in O_{m} \quad \text { implies } \quad m(\gamma)>m, \\
N_{C_{1}}\left(f_{1}\right) \subseteq M(R, 1)
\end{gathered}
$$

$$
\begin{aligned}
& D_{1}:\left\{T_{\delta} \mid \delta \in D_{1, \gamma}, \gamma \in C_{1}\right\} \quad\left(D_{1, \gamma}=\left\{\delta \mid \delta \in A_{2 m(\gamma)}, T_{s} \subseteq T_{\gamma}\right\}\left(\gamma \in O_{1}\right)\right), \\
& N_{C_{2}}\left(f_{2}\right) \subseteq N_{D_{1}}\left(f_{1}\right) \cap M(R, 2) \\
& D_{2}:\left\{T_{\delta_{1}}\left\{\delta \in D_{2, \gamma}, \gamma \in C_{2}\right\} \quad\left(D_{2, \gamma}=\left\{\delta \mid \delta \in A_{2 m(\gamma)}, T_{\delta} \subseteq T_{\gamma}\right\}\left(\gamma \in C_{2}\right)\right\},\right.
\end{aligned}
$$

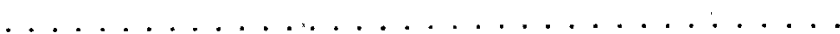

$$
\begin{aligned}
& N_{C_{h}}\left(f_{h}\right) \subseteq N_{D_{h-1}}\left(f_{h-1}\right) \cap M(R, h) \\
& D_{h}:\left\{T_{\delta} \mid \delta \in D_{h_{\gamma},}, \gamma \in C_{h}\right\} \quad\left(D_{h, y}=\left\{\delta \mid \delta \in A_{g m(\gamma)}, T_{\delta} \subset T_{\nu}\right\}\left(\gamma \in O_{h}\right)\right), \\
& \ldots \ldots \ldots \ldots \ldots \ldots \ldots \ldots \ldots
\end{aligned}
$$

Then, since $f_{h} \in N_{D_{k}}\left(f_{k}\right)(h \geqslant k)$, we have

$$
d\left(\pi f_{k}(p), \pi f_{h}(p)\right)<1 / 2 m(\gamma)<1 / k \quad(h \geqslant k)
$$

for some $\gamma \in C_{k}$, and hence $\left\{\pi t_{h}(p) \mid h=1,2, \ldots\right\}$ uniformly converges to a continuous mapping $\Phi(p)$ of $R$ into $I_{n+1}$.

Let us show that

$$
(c(p), \Phi(p))=\varphi(p) \epsilon \bigcap_{m=1}^{\infty} M(R, m) .
$$

Since $f_{h} \in N_{D_{k}}\left(f_{k}\right) \quad(h \geqslant k)$, if we take, for given $\gamma \in O_{k}$ and $p \in S_{\gamma}$, $\delta \in D_{k, \gamma}: p \in S_{\delta}$, then

hence

$$
d\left(\pi f_{h}(p), \pi f_{k}(p)\right)<1 / 2 m(\gamma) \quad(h \geqslant k)
$$

$$
d\left(\Phi(p), \pi f_{k}(p)\right) \leqslant 1 / 2 m(\gamma)<1 / m(\gamma) \quad\left(p \in S_{\gamma}\right)
$$

Therefore

$$
p(p) \in N_{C_{k}}\left(f_{k}\right) \subseteq M(R, k), \quad i, e ., \quad p(p) \in \bigcap_{m=1}^{\infty} M(R, m)
$$

Thus we get a homeomorphic mapping $\varphi$ of $R$ into $N(\Omega) \times I_{2 n+1}$.
COROILARY 4. Let $R$ be a metric space having the local Lindeloff propenty $\left({ }^{20}\right)$ such that $\operatorname{dim} R \leqslant n$. Then $R$ is homeomorphic to a subset of $N(\Omega) \times I_{2 n+1}$.

Proof. Since every metric space with the local Lindelöff property has a $\sigma$-star'-countable basis, this proposition is a direct consequence of Theorem 9 .

Theonerr 10. In order that a metric space $R$ with a $\sigma$-star-finite (countable) basis have dimensions $\leqslant n$ and have an open basis whose cardinal number is not greater than 1 it is necessary and sufficient that $R$ be homeomorphic with a subset of $N(\Omega) \times M I_{2 n+1}^{n}$, where $\Omega$ is a set with $|\Omega|=\mathrm{m}$, and $M_{2 n+1}^{n_{n+1}}$ is the set of points in $I_{2 n+1}$ at most $n$ of whose coordinates are rational.

Proof. Since it is well known that $\operatorname{dim} M_{2 n+1}^{n}=n, \operatorname{dim} N(\Omega) \times M_{2 n+1}^{n}=n$ from the generalized product theorem (see [\$] and [5]). Hence the sufficiency is obvious.

The proof of the necessity is analogous to that of Theorem 9. Let $L_{1}, L_{2}, \ldots$ be a sequence of $n$-dimensional linear subspaces in $I_{2 n+1}$; then we shall prove generally that $R$ is homeomorphie with a subset of $N(\Omega) \times\left(I_{2 n+1}-\bigcup_{n=1}^{\infty} L_{n n}\right)$. If $L_{1}, L_{2}, \ldots$ are all the linear spaces in $I_{2 n+1}$ of the form $x_{i_{1}}=r_{1}, \ldots, x_{i_{n+1}}=r_{n+1}$, the $r^{\prime} \mathrm{s}$ being rational, then we get the necessity part of this proposition. To show this we generally use the same notation as the above, but we replace $M(R, m)$ in the above proof by

$$
\begin{aligned}
& N(R, m)=\left\{\varphi \mid \varphi \in M(R), \varphi^{-1}\left(\mathfrak{I}_{\gamma}\right)<\mathfrak{H}_{m}(\gamma \in C),\right. \\
& \left.\pi \overline{\left(S_{\gamma}\right)} \cap L_{n}=\varnothing(\gamma \in O) \text { for some star-decomposition } C\right\} \text {. }
\end{aligned}
$$

The part 1 of the abore proof (of Theorem 9) is suitable for the present proof too.

We now prove $N_{C}(f) \cap N(R, m) \neq \emptyset$ for every $f \in M(R)$, every stardecomposition $O$ and every positive integer $m$. We define $D_{\gamma}(\gamma \in C)$ and $\mathfrak{B}_{\delta}\left(\delta \in D_{\gamma}, \gamma \in C\right)$ in the same way as in the proof of Theorem $9\left({ }^{21}\right)$ and consider fixed $\gamma \in O$ and $\delta \in D_{\gamma}$. Assume that $V_{1}, \ldots, V_{s}$ are all the members of $\mathfrak{B}_{b}$. Then we select vertices $x\left(V_{i}\right)(i=1, \ldots, s)$ in $I_{2 n+1}$ and $p_{0}, p_{1}, \ldots, p_{n}$ in $L_{m}$ for which it is true that $d\left(\pi f\left(V_{i}\right), x\left(V_{i}\right)\right)<1 / 3 m(\gamma)$, the $x\left(V_{i}\right)$ and $p_{j}$ are in a general position in $E_{2 n+1}$. Defining $\varphi(p) \in M(R)$ by (C) in the above proof, we see $p \in N_{C}(f)$ in the same way.

${ }^{20}$ We mean by Lindelöff property the property that every open covering has countable subcovering. If every point of $R$ has a nbd whose closure has the Lindelöf property, then $R$ is said to have the local Lindelöf property.

(21) From now on we ornit "in the proof of Theorem 9 " for brevity. 
To show that $\varphi \in N(R, m)$ we consider fixed $\gamma \in O$ and $\delta \in D_{\gamma}$ and suppose that $\nabla_{i_{1}}, \ldots, \nabla_{i_{t}}$ are all the members of $\mathfrak{V}_{s}$ containing a given point $p$ of $S_{\delta}$. We denote by $L_{\delta}(p)$ the linear $(t-1)$-space in $I_{2 n+1}$ spanned by the vertices $\infty\left(V_{i_{1}}\right), \ldots, x\left(V_{i_{l}}\right)$. Then $\Phi_{\delta}(p) \in L_{\delta}(p) \subseteq I_{2 n+1}-L_{m}$ and there exists $h(\delta)>0$ such that $L_{\delta}(p) \cap L_{\delta}\left(p^{\prime}\right)=\emptyset$ implies $a\left(L_{\delta}(p), L_{\delta}\left(p^{\prime}\right)\right) \geqslant 2 / h(\delta)$. Defining a star-decomposition $E$ in the same way, we have $\varphi^{-1}\left(\mathfrak{I}_{8}\right)<\mathfrak{U}_{m}$ $(\varepsilon \in E)$ and

$$
\overline{\pi \varphi\left(S_{\varepsilon}\right)}=\overline{\Phi_{\delta}\left(S_{s}\right)} \subseteq \bigcup\left\{L_{\delta}(p) \mid p \in S_{\delta}\right\} \subseteq I_{2 n+1}-I_{m},
$$

proving $\varphi \in N(R, m)$

Next, in order to show that for every $\varphi \in N_{C}(f) \cap N(R, m)$ there exists a star-decomposition $\sigma^{\prime}$ satisfying $N_{C^{\prime}}(\varphi) \subseteq N_{C}(f) \dot{\sim} N(R, m)$, we shall prove $N_{E}(\varphi) \subseteq N(R, m)$ for some star-decomposition $E$. Since $\varphi \in N(R, m)$,

$$
\varphi^{-1}\left(\mathfrak{I}_{\beta}\right)<\mathfrak{H}_{m} \quad(\beta \in B), \quad \delta\left(\overline{\pi \varphi\left(\hat{S}_{\beta}\right)}, L_{m}\right) \geqslant 1 / l(\beta)>0 \quad(\beta \in B)
$$

for some star-decomposition $B$ and positive integers $l(\beta)(\beta \in B)$. Letting

$$
\max (2 m(\beta), l(\beta))=k(\beta), \quad E_{\beta}=\left\{\varepsilon \mid \varepsilon \in A_{l(\beta)}, T_{\theta} \subseteq T_{\beta}\right\} \quad(\beta \in B),
$$

we have a star-decomposition $E:\left\{S_{\varepsilon} \mid \varepsilon \in E_{\beta}, \beta \in B\right\}$. For an arbitrary $\psi \in N_{E}(\varphi) \quad \psi^{-1}\left(\mathfrak{I}_{\varepsilon}\right)<\mathfrak{U}_{m} \quad(\varepsilon \in E)$ is proved in the same way. Moreover $p \in S_{\varepsilon}$ implies

$$
a(\pi \psi(p), \pi \varphi(p))<\mathbf{1} / k(\beta)-\eta(\varepsilon) \leqslant 1 / l(\beta)-\eta(\varepsilon)
$$

for some $\eta(\varepsilon)>0$. Therefore

$$
a\left(\pi \psi\left(S_{\varepsilon}\right), L_{m}\right) \geqslant \eta(\varepsilon)>0 \quad(\varepsilon \in E),
$$

which means $\overline{\pi \psi\left(S_{\varepsilon}\right) \cap L_{m}}=\emptyset(\varepsilon \in E)$. Hence $\psi \in N(R, m)$, i. e., $N_{E}(\varphi)$ $\subseteq N(R, m)$. Thus we can conclude that $N_{C^{\prime}}(\varphi) \subseteq N_{C}(f) \cap N(R, m)$ for $C^{\prime}=D \wedge E$. Since we can prove $\bigcap_{m=1}^{\infty} N(R, m) \neq \emptyset$ in the same way, we have $\varphi(p) \epsilon \bigcap_{m=1}^{\infty} N(R, m)$ which topologically maps $R$ into $N(\Omega) \times M_{2 n+1}^{n}$.

Defrninion. We say that the $p$-dimensional density of a subset $S$ of a metric space is zero if and only if for every $\varepsilon>0$ there exists a decomposition $B=\bigcup\left\{A_{i, \gamma} \mid \gamma \in C, i=1,2, \ldots\right\}$ such that $\delta\left(A_{i, \gamma}\right)<\varepsilon\{\gamma \in C$, $i=1,2, \ldots), \sum_{i=1}^{\infty}\left[\delta\left(A_{i, \gamma}\right)\right]^{p}<\varepsilon(\gamma \in C)$ and such that $\bigcup_{i=1}^{\infty} A_{i, \nu}=S_{\gamma}$ is open in $S$ for every $\gamma \in O$ and $S_{\gamma^{\prime}} \cap S_{\gamma^{\prime}}=\varnothing\left(\gamma \neq \gamma^{\prime}\right)\left({ }^{22}\right)$.

(2s) This notion and the following theorems are deeply related with Hausdorff's $p$-dimensional measure and Szpilrajn's theorem respectively. See [7]. We denote by $\delta(A)$ the diameter of $A$.
THEOREM 11. Every metric space $R$ of $(n+1)$-density zero has dimension $\leqslant n(23)$.

Proof. Let us show that $\operatorname{ind} \operatorname{dim} R \leqslant n$. We consider an arbitrary pair $F, G$ of closed sets with $\varrho(F, G)>0$. If we can show the existence of an open set $U$ with $\operatorname{dim}(\bar{U}-U) \leqslant n-1$, then ind $\operatorname{dim} R \leqslant n$ is proved. ( $\left.{ }^{24}\right)$. Select a positive integer $m$ with $1 / m<\varrho(F, G)$, and let $R=\bigcup\left\{A_{i, \gamma} \mid \gamma \in C, i==1,2, \ldots\right\}$ be a decomposition of $R$ such that

$$
\delta\left(A_{i, \gamma}\right)<1 / m^{2}, \quad \sum_{i=1}^{\infty}\left[\delta\left(A_{i, \gamma}\right)\right]^{n+1-1}<1 / m^{2}
$$

and such that $S_{\gamma}=\bigcup\left\{A_{i, y} \mid i=1,2, \ldots\right\}$ is open for every $\gamma \in O$. We put

$$
u_{i, \gamma}=\sup \left\{\varrho(F, x) \mid x \in A_{i, y}\right\}, \quad v_{i, \gamma}=\inf \left\{\varrho(F, x) \mid x \in A_{i, \gamma}\right\} .
$$

Then it is easily seen that $u_{i, \gamma}-v_{i, \gamma} \leqslant \delta\left(A_{i, \gamma}\right)$. We define a non-negatively valued function $d_{r}(r)$ for every $\gamma \in C$ by

It follows from

$$
\begin{aligned}
& d_{i, \gamma}(r)=\left\{\begin{array}{cl}
0 & \left(0 \leqslant r<v_{i, \gamma} \text { or } u_{i, \gamma}<r\right) \\
{\left[\delta\left(A_{i, \gamma}\right)\right]^{n}} & \left(v_{i, \gamma} \leqslant r \leqslant u_{i, \gamma}\right),
\end{array}\right. \\
& d_{\gamma}(r)=\sum_{i=1}^{\infty} a_{i, \gamma}(r) .
\end{aligned}
$$

that

$$
\int_{0}^{1 / m} a_{i, \gamma}(r) d r \leqslant\left[\delta\left(A_{i, \gamma}\right)\right]^{n+1}
$$

$$
\int_{i}^{1 / m} a_{\gamma}(r) d r=\int_{0}^{1 / m} \sum_{i=1}^{\infty} d_{i, \gamma}(r) d r=\sum_{i=1}^{\infty} \int_{0}^{1 / m} d_{i, \gamma}(r) d r \leqslant \sum_{i=1}^{\infty}\left[\delta\left(A_{i, \gamma}\right)\right]^{n+-1}<1 / m^{2}
$$

since considering $d_{i, \gamma}(r) \geqslant 0$ we may interchange integration and summation by Lebesgue's theorem. This implies $d_{r}(r(\gamma))<1 / m$ for some $r(\gamma)$ with $0<r(\gamma) \leqslant 1 / m$. We denote by $S(F, r)$ the set of all the points satisfying $\varrho(F, x)<r$ and by $S(r)$ the boundary of $S(F, r)$. Then $\left[\delta\left(A_{i, \gamma} \cap S(r(\gamma)) \mid\right]^{n} \leqslant d_{i, \gamma}(r(\gamma))\right.$ combined with $a_{\nu}(r(\gamma))<1 / m$ implies $\sum_{i=1}^{\infty}\left[\delta\left(A_{i, \gamma} \cap S(r(\gamma))\right)\right]^{n}<1$. $m$. We notice that $\bigcup\left\{S_{\gamma} \cap S(F, r(\gamma)) \mid \gamma \in O\right\}=U$

(2a) This theorem is an extension of Szpilrajn's theorem "every metric space of $(n+1)$-measure zero has $\operatorname{dim} \leqslant n$ to a non-separable case". See [7].

$\left.{ }^{24}\right)$ ind $\operatorname{dim} R \leqslant n$ if and only if $R$ has a $\sigma$-locally finite open basis $u$ such that the boundary of each set of $\mathfrak{u}$ has ind dim $\leqslant n-1$. See [5]. 
is evidently an open set of $R$ satisfying $F \subseteq U \subseteq G^{c}$. Since $\bar{U}-U$ $=\bigcup\left\{A_{i, y} \cap S(r(\gamma)) \mid \gamma \in C, i=1,2, \ldots\right\}$, the $n$-dimensional density of $\vec{U}-U$ is zero. The above arguement is also valid for $n=0$; hence $\bar{U}-U=\emptyset$ for a space $R$ of 1 -dimensional density zero, proving ind dim $R \leqslant 0$. Thus we can inductively establish this theorem.

THEOREM 12. If a metric space $R$ has dimension $\leqslant n$ and has a $\sigma$-star. finite (countable) basis, then it is homeomorphic to a subset $S$ of $N(\Omega) \times I_{2 n+1}$ such that $(n+1)$-density of $S$ is zero.

Proof. We define the distance $d^{\prime \prime}(x, y)$ between two points $x=\left(x_{1}, x_{2}\right)$, $y=\left(y_{1}, y_{2}\right)$ of $N(\Omega) \times I_{2 n+1}$ for $x_{1}, y_{1} \in N(\Omega), x_{2}, y_{2} \in I_{2 n+1}$ by $d^{\prime \prime}(x, y)$ $=d^{\prime}\left(x_{1}, y_{1}\right)+d\left(x_{2}, y_{2}\right)$. Replacing $M(R, m)$ in the proof of Theorem 9 by

$O(R, m)=\left\{\varphi \mid \varphi \in M(R), \varphi^{-1}\left(\mathfrak{I}_{y}\right)<\mathfrak{U}_{n i}(\gamma \in O)\right.$ for some star-decomposition $O$ and there exist decompositions $\overline{\varphi(R)} \cap T_{\gamma}=\bigcup_{i=1}^{\infty} A_{i, \gamma}(\gamma \in C)$ such that

$$
\left.\delta\left(A_{i, \gamma}\right)<1 / m, \sum_{m=1}^{\infty}\left[\delta\left(A_{i, \gamma}\right)\right]^{n+1}<1 / m(\gamma \in C, i=1,2, \ldots)\right\},
$$

we can analogousiy prove $\bigcap_{m=1}^{\infty} O(R, m) \neq \varnothing$. Part 1 of the proof of Theorem 9 is suitable for the present proof.

To prove $N_{C}(f) \cap O(R, m) \neq \emptyset$ for every $f \in M(R)$, every star-decomposition $O$ and every positive integer $m$, we define $\varphi \in M(R)$ by (C) in the proof of Theorem 9. Since $\pi\left(\varphi(R) \cap T_{\delta}\right)=\pi \varphi\left(S_{\delta}\right)$ for a fixed $\delta$ is contained in an $n$-dimensional polytope in $I_{2 n+1}, \pi\left(\overline{\varphi(R)} \cap T_{\delta}\right)$ is also contained in an $n$-dimensional polytope because.

$$
\pi\left(\overline{\varphi(R)} \cap T_{\delta}\right) \subseteq \pi \overline{\varphi\left(S_{\delta}\right)} \subseteq \overline{\pi \varphi\left(S_{\delta}\right)}
$$

It is well-known that the $(n+1)$-dimensional measure of an $1 k$-dimensional polytope is zero (see [7]), and hence, by the compactness of $\pi\left(\overline{\varphi(R)} \cap T_{\delta}\right)$, there exist open sets $K_{i, \delta}(i=1,2, \ldots, p(\delta))$ of $I_{2 n+1}$ such that

$$
\pi\left(\overline{\varphi(R)} \cap T_{\delta}\right) \subset \bigcup_{i=1}^{p(\delta)} \pi_{i, \delta}, \quad \delta\left(K_{i, \delta}\right)<1 / m, \quad \sum_{i=1}^{p(\delta)}\left[\delta\left(K_{i, b}\right)\right]^{n+1}<1 / m .
$$

We can select a positive integer $h(\delta)$ satisfying

$$
\begin{gathered}
\sum_{i=1}^{p(t)}\left[\delta\left(K_{i, \delta}^{r}\right)+1 / h(\delta)\right]^{n+1}<1 / m, \quad \delta\left(K_{i, \delta}\right)+1 / h(\delta)<1 / m \quad(i=1, \ldots, p(\delta)) . \\
L(p) \cap L\left(p^{\prime}\right)=\varnothing \quad \text { implies } \quad l\left(L(p), L\left(p^{\prime}\right)\right) \geqslant 2 / h(\delta) .
\end{gathered}
$$

Then we consider a star-decomposition

$$
E:\left\{S_{\varepsilon} \mid \varepsilon \in E_{\delta}, \delta \in D_{\gamma}, \gamma \in O\right\} \text { for } E_{\delta}=\left\{\varepsilon \mid \varepsilon \in A_{h(\delta)}, T_{\varepsilon} \subset T_{\delta}\right\}
$$

$p^{-1}\left(\mathfrak{I}_{k}\right)<\mathfrak{U}_{m}$ is proved in the same way. Horeover it follows from

that

$$
\delta\left(T_{c}\right) \leqslant \frac{1}{h(\delta)+1}
$$

$$
\begin{gathered}
\delta\left(T_{e} \times K_{i, n}\right) \leqslant \delta\left(K_{i, \delta}\right)+1 / h(\delta)<1 / m \\
\sum_{i=1}^{p(\delta)}\left[\delta\left(T_{e} \times K_{i, \delta}\right)\right]^{n+1} \leqslant \sum_{i=1}^{p(\delta)}\left[\delta\left(K_{i, \delta}\right)+1 / h(\delta)\right]^{n+1}<1, m .
\end{gathered}
$$

Since $\overline{\varphi(R)} \cap T_{\varepsilon} \subseteq \bigcup_{i=1}^{p(\delta)}\left(T_{\varepsilon} \times K_{i, \delta}\right)$ is obvious, we have $\varphi \in O(R, m)$.

Next let us prove that $\varphi \in N_{C}(f) \cap O(R, m)$ implies $N_{C^{\prime}}(\varphi) \subset N_{C^{\prime}}(f)$ $\cap O(R, m)$ for a suitable star-decomposition $G^{\prime}$. We have, by $\varphi \in \widehat{O(R, m)}$, a star-decomposition $B$ such that $\varphi^{-1}\left(\mathfrak{T}_{\beta}\right)<\mathfrak{U}_{m}$ and such that

$$
\overline{\varphi(R)} \cap T_{\beta}=\bigcup_{i=1}^{\infty} A_{i, \beta}, \quad \delta\left(A_{i, \beta}\right)<1 / m, \quad \sum_{i=1}^{\infty}\left[\delta\left(A_{i, \beta}\right)\right]^{n+1}<1 / m
$$

for some $A_{i, \beta}$. This implies

$$
(\beta \in B, i=1,2, \ldots)
$$

$$
\begin{aligned}
\pi\left(\overline{\varphi(R)} \cap T_{\beta}\right)=\bigcup_{i=1}^{\infty} \pi\left(A_{i, \beta}\right), \quad \delta\left(\pi\left(A_{i, \beta}\right)\right)<1 / m, & \sum_{i=1}^{\infty}\left[\delta\left(\pi\left(A_{i, \beta}\right)\right)\right]^{n+1}<1 / m \\
& (\beta \in B, i=1,2, \ldots) .
\end{aligned}
$$

Hence, by the compactness of $\pi\left(\overline{\varphi(R)} \cap T_{f}\right)$, there exist open sets $H_{i, \beta}$ $(\beta \in B, i=1, \ldots, q(\beta))$ of $I_{2 n+1}$ satisfying

$$
\begin{aligned}
& \pi\left(\overline{\varphi(R)} \cap T_{\beta}\right) \subset \bigcup_{i=1}^{q(i)} H_{i, \beta}, \quad \delta\left(H_{i, \beta}\right)<1 / m, \quad \sum_{i=1}^{q(\beta)}\left[\delta\left(H_{i, \beta}\right)\right]^{n+1}<1 ! m \\
&(\beta \in B, i=1, \ldots, q(\beta)) .
\end{aligned}
$$

We choose a positive integer $h(\beta)$ for every $\beta \in B$ satisfying

$$
\sum_{i=1}^{q(\beta)}\left[\delta\left(H_{i, \beta}\right)+5 / h(\beta)\right]^{n+1}<1 / m, \quad \delta\left(H_{i, \beta}\right)+5, h(\beta)<1 / m
$$

$$
(i=1, \ldots, q(\beta))
$$


Letting

$$
k(\beta)=\max (2 m(\beta), h(\beta)), \quad E_{\beta}=\left\{\varepsilon \mid \varepsilon \in A_{k(\beta)}, T_{\varepsilon} \subseteq T_{\beta}\right\} \quad(\beta \in B)
$$

we have a star-decomposition $E:\left\{S_{\mathrm{s}} \mid \varepsilon \in E_{\beta}, \beta \in B\right\}$.

To prove $N_{E}(\varphi) \subseteq O(R, m)$, we consider a given $\psi \in N_{x}(\varphi)$. Then $\psi^{-1}\left(\mathfrak{I}_{z}\right)<\mathfrak{H}_{m}(\varepsilon \in E)$ is proved in the same way. On the other hand, for any $x \in \overline{\psi(R)} \cap T_{\varepsilon}$ there exists $y \in \psi(R) \cap T_{\varepsilon}=\psi\left(S_{\varepsilon}\right)$ with $d(\pi(x), \pi(y))$ $<1 / h(\beta)$. Let $\psi(\beta)=y, p \in S_{\varepsilon}$; then it follows from

$$
d(\pi \psi(p), \pi \varphi(p))<1 / k(\beta) \leqslant 1 / h(\beta)
$$

for $\beta \in B$ with $\varepsilon \in E_{\beta}$ that $d(\pi(x), \pi \varphi(p))<2 / h(\beta)$. That is to say for any $x \in \overline{\psi(R)} \cap T_{\varepsilon}$ we can select $z \in \varphi(k) \cap T_{\varepsilon}$ satisfying $d(\pi(x), \pi(x))$ $<2 / h(\beta)$. Hence letting

$B_{i, \varepsilon}=\left\{x \mid x \in \overline{\psi(R)} \cap T_{\varepsilon}, d(\pi(x), \pi(z))<2 / h(\beta)\right.$ for some $\left.\pi(z) \in H_{i, \beta}\right\}$

we have $\overline{\psi(R)} \cap T_{\varepsilon}=\bigcup_{i=1}^{q(\beta)} B_{i, \varepsilon}$. For given $x_{1}, x_{2} \in B_{i, 8}$ we take $z_{1}, z_{2}$ with

$$
d\left(\pi\left(x_{1}\right), \pi\left(z_{1}\right)\right)<2 / h(\beta), \quad d\left(\pi\left(x_{2}\right), \pi\left(z_{2}\right)\right)<2 / h(\beta), \quad \pi\left(z_{1}\right), \pi\left(z_{2}\right) \in H_{i, \beta} .
$$

Therefore $d\left(\pi\left(x_{1}\right), \pi\left(x_{2}\right)\right)<\delta\left(H_{i, \beta}\right)+4 / h(\beta)$, which implies $d^{\prime \prime}\left(x_{1}, x_{2}\right)$ $<\delta\left(H_{i, \beta}\right)+5 / h(\beta)$ since $x_{1}, x_{2} \in X_{\varepsilon}$. Thus we have

$$
\begin{gathered}
\delta\left(B_{i, \varepsilon}\right) \leqslant \delta\left(H_{i, \beta}\right)+5 / h(\beta)<1 / m, \\
\sum_{i+1}^{q(\beta)}\left[\delta\left(B_{i, \delta}\right)\right]^{n+1} \leqslant \sum_{i=1}^{q(\beta)}\left[\delta\left(H_{i, \beta}\right)+5 / h(\beta)\right]^{n+1}<1 / m
\end{gathered}
$$

proving $\psi \in O(R, m)$, i. e., $N_{E}(\varphi) \subseteq O(R, m)$. This combined with $N_{D}(\varphi)$ $\subset N_{C}(f)$ for a suitable $D$ implies $\bar{N}_{O^{\prime}}(\varphi) \subset N_{C}(f) \cap O(R, m)$ for $C^{\prime}=D \wedge E$. Since we can prove $\bigcap_{m=1}^{\infty} O(R, m) \neq \varnothing$ in the same way, we get $\varphi(p) \epsilon \bigcap_{m=1}^{\infty} O(R, m)$, which topologically maps $R$ on $\varphi(R)$ of $(n+1)$-dimensional density zero.

\section{References}

[I] P. Alexandroff and A. Kolmogoroff, Endliche Utberdeckungen topologischer Räume, Fund. Math. 26 (1936), p. 267-271.

[2] W. Hurewicz and H. Wallman, Dimension theory, 1941.

[3] J. de Groot and H. de Vries, A note on non-Arehimedean metrization, Proceedings Koninkl. Nederl. Akademie van Wetenschappen, Ser. A, 58, No. 2 (1955), p. 222-224.

[4] M. Katětov, On the dimension of non-separable spaees I, Czechoslovak Mathematical Journal 2 (77) (1952), p. 338-368.
[5] K. Morita, Normal families and dimension theory for metrie spaess, Math. Annalen 128 (1954), p. 350-362

[6] A. H. Stone, Paracompactness and product spaces, Bull. Amer. Math. Soc., 54 (1948), p. 977-982.

[7] E. Szpilrajn, La dimension et la mesure, Fund. Math. 28 (1937), p. 81-89.

[8] J. W. Tukey, Oonvergence and uniformity in topology, 1940.

OSAKA CITY UNIVERSITY

\section{Reou par la Rédaction le 12.4.1957}

Addendum. Recently we have proved by applying Theorem 3 that every metrie space can be topologioally imbedded in a product of an enumerable number of metric spaces $R_{i}$ of $\operatorname{dim} R_{i} \leqslant 1 \quad(i=1,2, \ldots)$. See $O n$ imbedding a metric space in a product of one-dimensional spaces, Proc. of Japan Acad. 33 (1957), p. 445-449. 